\title{
A Structural Model of the Retail Market for Illicit Drugs*
}

\author{
Manolis Galenianos $\quad$ Alessandro Gavazza ${ }^{\S}$ \\ September 2016
}

\begin{abstract}
We estimate a model of illicit drugs markets using data on purchases of crack cocaine. Buyers are searching for high-quality drugs, but they determine drugs' quality (i.e., their purity) only after consuming them. Hence, sellers can rip off first-time buyers or can offer higher-quality drugs to induce buyers to purchase from them again. In equilibrium, a distribution of qualities persists. The estimated model implies that if drugs were legalized, in which case purity could be regulated and hence observable, the average purity of drugs would increase by approximately 20 percent and the dispersion would decrease by approximately 80 percent. Moreover, increasing penalties may raise the purity and affordability of the drugs traded by increasing sellers' relative profitability of targeting loyal buyers versus first-time buyers.
\end{abstract}

*We are grateful to the Editor, three anonymous referees, Nicola Pavoni and many seminar audiences for useful comments and discussions. Mi Luo and Simon Mongey provided excellent research assistance.

IDepartment of Economics, Royal Holloway, University of London. Egham Hill, Egham TW20 0EX, United Kingdom. Email: manolis.galenianos@gmail.com.

$\S$ Department of Economics, London School of Economics. Houghton Street, London WC2A 2AE, United Kingdom. Email: a.gavazza@lse.ac.uk. 


\section{Introduction}

How do markets for illicit commodities, such as narcotics, differ from regular markets? What would happen to the consumption and prices of narcotics if their trade were legalized? How do changes in the intensity of enforcement affect them?

The goal of this paper is to provide quantitative answers of these questions. With this goal in mind, we estimate a model that focuses on pervasive moral hazard as the distinguishing characteristic of the retail market for illicit drugs - i.e., the "cutting" of drugs. ${ }^{1}$ We quantify the effects of sellers' moral hazard on the quality and consumption of drugs, providing potential insights into how market outcomes might differ under legalization. Our quantitative analyses suggest that the presence of moral hazard leads to counter-intuitive implications for policing.

We model a market with unobserved quality, search frictions and repeated trade. Buyers with heterogeneous tastes for drugs search for sellers with heterogeneous costs of supplying drugs. Buyers meet new sellers randomly and cannot observe drug purity before consuming it-i.e., illicit drugs are experience goods. Following the key insight of Galenianos et al. (2012), quality is non-contractible, which creates a moral hazard problem; this is the key way in which the model captures an illegal market in which there are no institutions to enforce quality standards. ${ }^{2}$ When a buyer meets a seller who offers a sufficiently high level of quality, he forms a long-term relationship with this seller. The buyer-seller relationship persists until either the buyer meets a new seller who offers higher-quality drugs or an exogenous shock (e.g., policing) severs it.

Buyers' inability to ascertain drug quality creates a trade-off for sellers. On the one hand, moral hazard: sellers can offer low-quality drugs, thereby maximizing instantaneous profits. On the other hand, long-term relationships: sellers can offer high-quality drugs that induce buyers to purchase from them again, thereby increasing their customer base. Hence, long-term relationships are the principal counterweight to the moral hazard problem inherent in illegal markets. In equilibrium, a distribution of quality levels persists: high-cost sellers rip their (firsttime) buyers off by offering zero-purity drugs, whereas low-cost sellers offer high-quality drugs to attract long-term customers.

We estimate the model combining two distinct datasets that provide key pieces of information on the crack cocaine market in the U.S.: 1) the distribution of drug qualities offered in the

\footnotetext{
${ }^{1}$ While some legal markets feature quality uncertainty and sellers' moral hazard, in Section 3, we document that approximately 15 percent of crack cocaine purchases involve zero purity-i.e., they are complete rip-offs. It is hard to find a legal market with comparable levels of outright fraud. Moreover, the cutting of drugs happens mainly at the retail level since wholesale operations (i.e., transportation) are more efficient if drugs are pure.

${ }^{2}$ We study the market given that trade is illegal, abstracting from why it is illegal, which may be because of paternalism or because of externalities that market participants impose on non-market participants (for an analysis of some of the externalities due to crack cocaine, see Fryer et al., 2013). Hence, we do not perform a welfare analysis in the counterfactuals of Section 5.4 since, for example, externalities could be of first-order importance in aggregate welfare. Of course, some of these externalities arise exactly because trade is illegal.
} 
market; 2) the proportion of buyers who purchase from their regular sellers; and 3) buyers' frequency of drug purchases, including the difference in purchase frequency between those who buy from regular sellers and those who buy from new sellers. The model fits the data well, and the estimation reveals that the key elements of our model play an important role in the crack cocaine market. Specifically, search frictions are non-trivial: a buyer meets a new seller, on average, approximately every 24 days. Relationships between buyers and sellers are quite short-lived: they exogenously end, on average, every 41 days. Nonetheless, regular buyers are valuable to sellers, as they consume very frequently - on average, approximately 19 times per month - confirming that frequent users account for the vast majority of crack cocaine purchases (Kilmer et al., 2014). Overall, search and information frictions imply a large dispersion of drug qualities. Finally, the estimates imply that sellers' profits are extremely skewed, with very few (low-cost) sellers reaping substantial profits and most sellers earning less than the minimum wage, consistent with the descriptive evidence reported by Reuter et al. (1990) and Levitt and Venkatesh (2000).

We use our parameter estimates to perform two counterfactual analyses. First, we quantify the importance of imperfect observability of drug quality and moral hazard for market outcomes. We modify our baseline model by assuming that buyers observe drug quality before making a purchase, thereby eliminating moral hazard. In the equilibrium of this counterfactual market, zero-purity drugs disappear, but quality dispersion persists due to search frictions. Quantitatively, price-adjusted average quality increases by 20 percent. Interestingly, the standard deviation of purity decreases by approximately 80 percent relative to the baseline case, implying that imperfect observability, rather than search frictions, is the main cause of the dispersion of quality. ${ }^{3}$ Overall, these large changes in the distribution of purity trigger smaller increases in the number of buyers (of seven percent) and in the individual consumption of crack cocaine (of four percent). Since we posit that pervasive imperfect observability and moral hazard are defining characteristics of illegal markets, this counterfactual could provide some insights into how outcomes would differ if drugs markets were legal. Of course, legalization would affect many other features of the market, but our analysis points out that quality uncertainty will decrease and the average quality of drugs will increase if buyers gain better information.

Our second counterfactual studies how penalties on buyers and sellers affect market outcomes. The motivation is that in the past 30 years the U.S. has markedly increased the enforcement and severity of its drug laws - the so-called "war on drugs" — which has resulted in the tripling of arrests for drug-related offenses. ${ }^{4}$ Interestingly, during the same period, drugs have become dramatically cheaper and purer. In our model, lower enforcement may lead to lower drug quality and

\footnotetext{
${ }^{3} \mathrm{~A}$ reduction in quality dispersion may bring health benefits: Caulkins (2007) argues that greater variability in purity predicts overdoses, because users inadvertently consume more pure drug than they intend to when they purchase drugs that are more pure than is typical.

${ }^{4}$ At the same time, the number of arrests for non-drug related offenses has barely changed (Kuziemko and Levitt, 2004).
} 
higher quality uncertainty. This counterintuitive result is mainly the outcome of the interaction between moral hazard and long-term relationships. Lower enforcement on sellers increases their number. Hence, the rate at which buyers meet with new sellers increases, and the expected duration of buyer-seller relationships declines. Therefore, the profitability of establishing long-term relationships falls relative to that of cheating, which leads to more cheating and lower average quality. In addition, the newly-entered sellers have higher costs and, thus, are more likely to rip off their customers.

Quantitatively, we find that a 15-percent decrease in sellers' penalties leads to a 13-percent decrease in average quality and a 20-percent increase in the standard deviation of quality (and similar results obtain when we reduce buyers' penalties). Hence, our analysis suggests that increasing penalties may have contributed to the observed increased purity and affordability of retail drugs in the U.S. Of course, the market for drugs has changed in many ways over time (among others, through economies of scale in the transportation of drugs to the U.S.); nonetheless, we find it interesting that our model is consistent with these observed trends.

The paper proceeds as follows. Section 2 reviews the literature. Section 3 introduces the data. Section 4 presents the theoretical model. Section 5 presents our quantitative analysis: we estimate the model, illustrate its main implications, and perform counterfactual analyses.

Section 6 concludes. Appendices A and B collect all proofs and report the derivation of the density of buyers' preferences in the ADAM dataset, respectively. Online Appendices C and D further present the details of the solution of the model with observable quality and report on additional counterfactual analyses, respectively.

\section{$2 \quad$ Related Literature}

The paper contributes to several strands of the empirical literature. The first, which is vast and mainly descriptive, is the literature on illegal markets. Most empirical analyses of illegal markets rely on the traditional economic assumptions of perfect information and/or a centralized market, following the influential theoretical analysis of Becker et al. (2006). This framework, however, cannot explain the extensive amount of cheating that we observe in the data because it abstracts from two defining features of illicit markets on which our quantitative analysis focuses: moral hazard and search frictions. Hence, it may not be well suited to understanding the implications of different policies, such as penalties on buyers and sellers. In this strand of the literature, the closest papers include Reuter and Caulkins (2004), which discusses information issues in drug markets; Jacobi and Sovinsky (2016), which studies buyers' limited information about drug accessibility; and Adda et al. (2014), which analyzes the effects of depenalization on the cannabis market (but does not consider the role of information frictions). We build on Galenianos et al. (2012), but we differ in two ways: our primary contribution is empirical, and 
we include some novel features in our model (recounted in Section 4). In turn, our framework delivers a closer connection with the data and allows us to perform out-of-sample analyses.

Our second contribution is to the literature that studies firms' quality decisions when quality is not observable (or not contractible). To our knowledge, papers in this strand of the literature examine markets for legal goods. Many empirical contributions have analyzed the role of quality certification and consumers' and suppliers' responses to it; for a thorough survey, see Dranove and Jin (2010). Empirical analyses of moral hazard have focused mainly on the behavior of intermediaries; see, for example, Iizuka (2012) for the case of physicians and prescription drugs. Our paper examines the importance of moral hazard in the context of an illegal market, where search and matching frictions are pervasive, and innovates on previous descriptive empirical work by estimating a dynamic equilibrium model that allows the quantification of these effects on market outcomes.

Finally, this paper is also related to the literature on the structural estimation of search models. Almost all empirical applications to product markets use static models: recent contributions include Hortaçsu and Syverson (2004), Hong and Shum (2006), Wildenbeest (2011), and Allen et al. (2014). Notable exceptions are Cebul et al. (2011), which uses a dynamic search model to understand the role of buyers' turnover in health insurance pricing, and Gavazza (forthcoming), which estimates a dynamic model of a decentralized asset market to study the effects of intermediaries on asset allocations and asset prices. Instead, we build a dynamic model that highlights the role of long-term buyer-seller relationships. ${ }^{5}$ Most applications of dynamic search models focus on labor markets; Eckstein and Van den Berg (2007) provide an insightful survey of this literature and, within the labor-market context, Bontemps et al. (1999) is the closest empirical paper. We innovate on all previous empirical contributions by quantifying the effects of an additional friction - i.e., the imperfect observability of product quality at the time of the transaction - and its implications for how penalties affect market outcomes.

\section{Data}

The available data dictate some of the modeling choices of this paper. For this reason, we describe the data before presenting the model. This description also introduces some of the identification issues that we discuss in more detail in Section 5.2.

\footnotetext{
${ }^{5}$ Hence, the paper also touches upon some of the issues that the literature on customer markets highlights. Most empirical investigations focus on markets for services, whereas we focus on a product market. See, for example, Boot (2000) for a survey of the literature on relationship banking.
} 


\subsection{Data Sources}

We combine two main datasets. The first is an extensive database on drug purchases; the second is a survey that collects information about drug use among arrestees. We now describe these dataset in more detail.

STRIDE. The System to Retrieve Information from Drug Evidence (STRIDE) is a database of drug exhibits sent to Drug Enforcement Administration (DEA) laboratories for analysis. Exhibits in the database are recorded by the DEA, other federal agencies, and local law enforcement agencies. The data contain records of acquisitions of illegal drugs by undercover agents and DEA informants. Economic analyses of markets for illegal drugs have widely used STRIDE, although it is not a representative sample of the drugs available in the United States. ${ }^{6}$

The entire dataset has approximately 915,000 observations for the period 1982-2007 for a number of different drugs and acquisition methods. We focus on crack cocaine and keep the observations acquired through purchases (i.e., we drop seizures) and clean the data of missing values and other unreliable observations, as Arkes et al. (2008) suggest. Our quantitative analysis in Section 5 uses data for the years 2001-2003 because of the time limitations of our other data source, described below. Moreover, since our model focuses on retail transactions, we include in our estimation sample purchases with a value of less than $\$ 200$ in real 2003 dollars.

ADAM. The Arrestee Drug Abuse Monitoring (ADAM) dataset is a quarterly survey of persons arrested or booked on local and state charges in various metropolitan areas in the United States. The survey asks questions about the use of drugs and alcohol. The arrestees participate in the survey voluntarily under full confidentiality: only about ten percent of the arrestees reject the interview request (Dave, 2008). In addition to the interviews, participants provide urine samples, which are analyzed for validation of arrestees' self-reported drug use. Since 2000, the survey includes a drug market procurement module, collecting information on the arrestee's number of drug purchases in the previous 30 days and whether or not the last purchase was from a regular dealer or not. ${ }^{7}$ We have data from the 2001-2003 surveys.

Since ADAM likely oversamples drug users, we complement these two main datasets with auxiliary data that allow us to account for selection into the ADAM sample. Specifically, the 2001-2003 National Survey on Drug Use and Health (NSDUH) provides survey data on the use of crack cocaine (as well as other illicit drugs) among the non-institutionalized U.S. population. Hence, while ADAM includes arrestees only, the NSDUH does not include them, thereby sampling

\footnotetext{
${ }^{6}$ Horowitz (2001) notes that the time series of drug prices in Washington, D.C. differ depending on which agency collected the data. However, Arkes et al. (2008) show that these inconsistencies disappear almost entirely by controlling for the size of the transaction (above or below five grams). Hence, we restrict our analysis to transactions with a value below 200 constant 2003 dollars.

${ }^{7}$ The possible answers also include an "occasional" dealer. In principle, we can explicitly incorporate this additional source into our model, and we conjecture that it would not alter sellers' trade-off between rip-offs vs. building a customer base. However, this extension would come at the cost of tractability.
} 
TABle 1: Summary Statistics

\begin{tabular}{|c|c|c|c|c|}
\hline PANEL A: STRIDE $(\mathrm{N}=2,321)$ & MEAN & Std. Dev. & MiN & Max \\
\hline PRICE (2003 DOLLARS) & 95.247 & 48.152 & 1.052 & 200.000 \\
\hline WEIGHT (GRAMS) & 1.120 & 1.118 & 0.004 & 9.500 \\
\hline PURITY $(\%)$ & 56.313 & 28.036 & 0.000 & 98.000 \\
\hline Pure Grams & 0.658 & 0.722 & 0.000 & 6.298 \\
\hline Pure Grams Per $\$ 100$ & 0.622 & 0.535 & 0.000 & 3.931 \\
\hline \multicolumn{5}{|l|}{ PANEL B: ADAM $(\mathrm{N}=64,462)$} \\
\hline ObTained CRaCk in Last 30 Days (\%) & 16.899 & 37.474 & 0.000 & 100.000 \\
\hline Purchased from Regular Dealer $(\%)$ & 52.481 & 49.941 & 0.000 & 100.000 \\
\hline Purchases in Past 30 Days, Matched & 16.331 & 11.124 & 1.000 & 30.000 \\
\hline Purchases in Past 30 Days, Unmatched & 11.548 & 10.419 & 1.000 & 30.000 \\
\hline \multicolumn{5}{|l|}{ Panel C: Auxiliary Data } \\
\hline CONSUMing CRACK IN NSDUH $(\%)$ & 0.800 & 8.908 & 0.000 & 100.000 \\
\hline ARREST RATe (\%) & 3.700 & - & 0.000 & 100.000 \\
\hline
\end{tabular}

Notes - This table provides summary statistics of the variables used in the empirical analysis. Panel A presents summary statistics of the variables obtained from the STRIDE dataset; Panel B presents summary statistics of the variables obtained from the ADAM dataset; Panel C presents summary statistics of our auxiliary variables. Drug prices have been deflated using the GDP Implicit Price Deflator, with 2003 as the base year.

the complement of the population sampled in the ADAM data. Furthermore, we obtain data on arrests from the Federal Bureau of Investigation and on the total U.S population from the U.S. Census to calculate the aggregate arrest rate of individuals over 15 years of age in $2002 .^{8}$

\subsection{Data Description}

Table 1 provides summary statistics of the main variables. Panel A refers to the STRIDE dataset, Panel B to the ADAM dataset, and Panel C to the auxiliary data.

Panel A reports some interesting patterns. Because most transactions happen at round dollar values (i.e., 50 or 100 dollars), the heterogeneity in PRICE is small relative to the heterogeneity in Pure Grams (i.e., the product of Weight and Purity): the coefficient of variation of Pure Grams is more than twice as large as that of Price. ${ }^{9}$ We take the ratio of Pure Grams

\footnotetext{
${ }^{8}$ Sources: http://www.fbi.gov/about-us/cjis/ucr/crime-in-the-u.s/2011/crime-in-the-u.s.2011/tables/table-32 and https://www.census.gov/popest/data/historical/2000s/vintage_2002/ index.html.

${ }^{9}$ Some variation in PRICE across years is due to the fact that we deflate prices.
} 


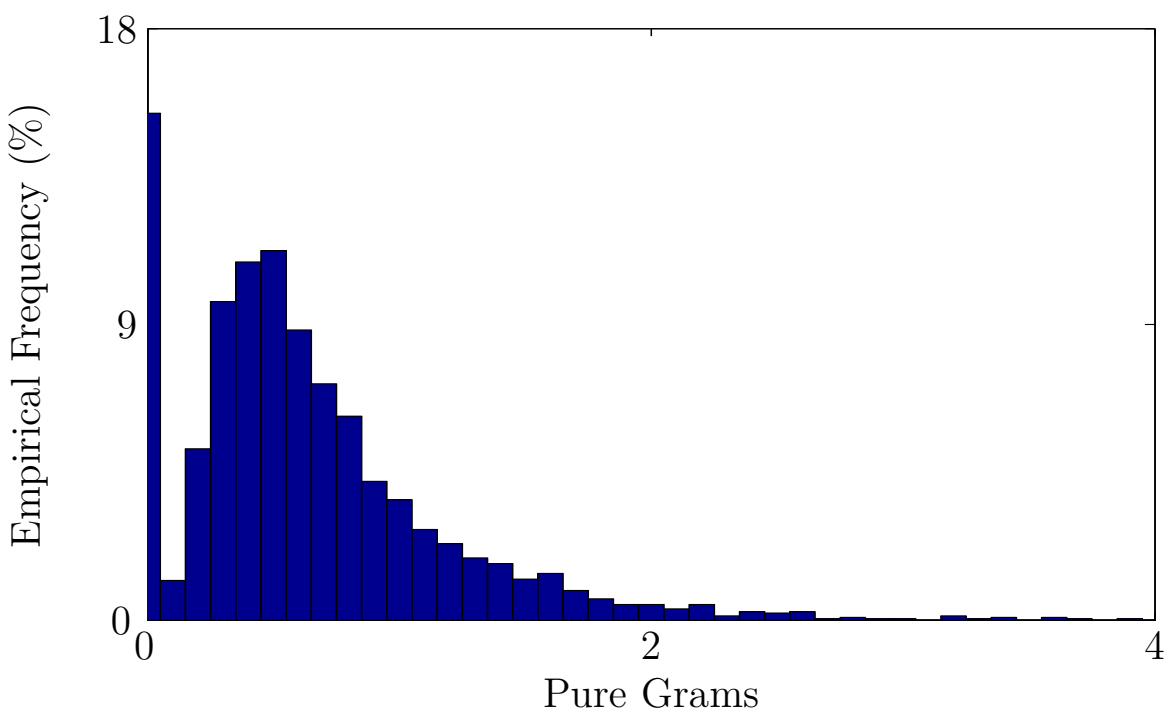

FIG. 1: Histogram of pure grams of crack cocaine per $\$ 100$.

and Price to construct the variable Pure Grams PER \$100; Figure 1 displays its empirical distribution, which shows substantial variation, with 15.4 percent of the observations having a value of zero-i.e., complete rip-offs. ${ }^{10}$ Moreover, there are almost no observations with a positive, but very small amount of crack cocaine, suggesting that the distribution features a gap between the mass point at zero and the density of positive qualities (Galenianos et al., 2012).

Panel B reports that approximately 17 percent of all arrestees purchased crack cocaine in the past 30 days. Among that group, the average number of PURCHASES IN PAST 30 DAYS equals 12.98 (thus, the unconditional average of PURChASES IN PAST 30 DAYS is 9.6), thereby confirming that frequent users account for the vast majority of drug expenditures (Kilmer et al., 2014). Of those who purchased crack cocaine, 52.5 percent report buying from their regular source. Interestingly, individuals purchasing from their regular dealers report an average of 16.3 Purchases IN PAst 30 DAYs, whereas individuals purchasing either from an occasional source or from a new source have an average of 11.5 PURCHASES IN PAST 30 DAYs (the $t$-statistics of the difference equals -21.39). The model will interpret this difference as different consumption rates between buyers who are currently matched to a seller and buyers who are currently not matched, taking into account that buyers' choice of matching with a seller will depend on their preferences for drugs.

Overall, these two main datasets provide a rich description of the retail crack cocaine market and are well-suited for investigating the role of search frictions, imperfect observability, and buyer-seller relationships in this market. Specifically, we take the distribution of PURE GRAMS PER $\$ 100$ displayed 1 as the distribution that first-time buyers face, and our model interprets its

\footnotetext{
${ }^{10}$ Galenianos et al. (2012) report that geographic and temporal variation is not large, contributing approximately 20 percent to the overall variation.
} 
dispersion as originating from both search frictions and imperfect observability, thereby allowing us to calculate the contribution of each to the overall dispersion. ${ }^{11}$ Moreover, the ADAM dataset is useful in measuring the frequency of buyer-seller long-term relationships and buyers' consumption rates. Furthermore, the auxiliary data reported in Panel C confirm that ADAM oversamples drug users, as the difference between the fraction of individuals consuming crack cocaine in the ADAM dataset and in the NSDUH dataset, respectively, is large; hence, these auxiliary data allow us to explicitly account for sample selection in the estimation. We should point out that, since we compute the arrest rate from aggregate data, this statistic has no sampling variability, which will affect the confidence intervals of the parameters that depend on this statistic; see Section 5.2.

Despite all of their advantages, however, the datasets pose some challenges. Most importantly, our datasets are cross-sectional, and, therefore, we do not observe buyers' and sellers' behavior over time. Specifically, we do not observe sellers transacting with several buyers or the dynamics of the terms of trade within individual buyer-seller relationships. These limitations imply that a model in which sellers discriminate between different buyers, while theoretically feasible, would be difficult to identify with the available data. Similarly, we do not observe whether sellers vary the quality of their offerings during their relationships with buyers, and theory argues that quality could either increase or decrease over time (Board and Meyer-ter Vehn, 2013). Hence, in the absence of a more-detailed measurement, our model (successfully) matches the available data by focusing on heterogeneity across sellers. Moreover, while the theory can accommodate multidimensional heterogeneity of buyers and/or sellers, our cross-sectional data make it difficult to identify such a model. Thus, we focus on a parsimonious framework with buyers' heterogeneity in their willingness to pay for drugs and sellers' heterogeneity in their costs of acquiring pure drugs, and we let other parameters be common across individuals. ${ }^{12}$ We discuss data limitations and their implications for our modeling assumptions further in Section 6.

\section{The Model}

We enrich the model of Galenianos et al. (2012) by introducing: 1) buyers' heterogeneity in their preferences for drugs; 2) sellers' heterogeneity in their costs of supplying drugs; and 3) a matching function that governs buyer-seller meetings. This richer framework allows the structural estimation to capture some key patterns of the data, such as the shape of the distribution of drug quality displayed in Figure 1. It also allows our counterfactual analyses to have some meaningful margins of adjustment in response to different environments, such as the entry (or exit) of buyers

\footnotetext{
${ }^{11}$ Given STRIDE's data collection, we believe that sales to regular buyers should account for a minimal share of the data, at most.

${ }^{12}$ The empirical labor search literature often makes similar assumptions that some parameters are homogeneous, as that literature faces data constraints similar to those in this paper.
} 
and of sellers, as well as their effects on buyers' and sellers' meeting rates.

\subsection{The Environment}

Time runs continuously; the horizon is infinite, and the future is discounted at rate $r$.

A continuum of potential buyers of measure $\bar{B}$ are characterized by a heterogeneous preference parameter $z$ that determines the instantaneous utility $z q$ of consuming drugs of quality $q .{ }^{13}$ Buyers' marginal utility $z$ is distributed according to a continuous, connected and log-concave distribution $\bar{M}(\cdot)$ with support $[0, \bar{z}]$. The heterogeneity in $z$ could arise because of differences in innate preferences or because of differences in addictions, as in Becker and Murphy (1988). ${ }^{14}$ Each buyer decides whether to participate in the market. If he does not participate, his payoff is zero; if he participates, he pays a flow cost $K_{B}$ and trades with sellers. Buyers' flow costs, like sellers' flow costs, discussed below, capture costs imposed on traders through reduced convenience, ethical constraints, and criminal punishments (Becker et al., 2006). Let $B$ be the measure of buyers who participate in the market and $M(\cdot)$ be the distribution of their types. Buyers maximize their expected discounted utility.

A continuum of potential sellers of measure $\bar{S}$ are characterized by their heterogeneous marginal cost $c$, which determines the cost $c q$ of providing drugs of quality $q$. Sellers' marginal cost $c$ is distributed according a continuous and connected distribution $\bar{D}(\cdot)$ with support $[0, \bar{c}]$. Seller heterogeneity aims to capture differences in the cost of acquiring pure drugs, perhaps because of differential connections with upstream suppliers. Each seller decides whether to participate in the market, in which case he pays a flow cost $K_{S} \cdot{ }^{15}$ We denote the measure of participating sellers by $S$ and the distribution of their types by $D(\cdot)$. These sellers maximize their steady-state profits.

The buyers and sellers who participate in the market meet and trade with each other. A buyer is either matched with a seller (his regular seller) or he is unmatched. There are two types of meetings: new meetings, where a buyer and a seller meet for the first time, and repeat meetings, where a buyer meets with his regular seller. Buyers choose whether or not to match with sellers, and sellers choose the quality $q$ that they offer to buyers. In more detail:

1. At a new meeting, the buyer pays a fixed price $p$ and receives drugs of quality $q$. The buyer does not observe the quality $q$ of the drugs he receives, but he learns it perfectly after consuming the drugs. After a new meeting, the buyer decides whether to form a match with the new seller. if the buyer is currently matched, this means that he severs his

\footnotetext{
13 "Quality" refers to a transaction's pure quantity.

${ }^{14}$ However, we do not model addiction over and above what buyers' entry decision captures.

${ }^{15}$ We assume that $\bar{c}$ is large enough so that sellers with the highest costs $\bar{c}$ do not participate in equilibrium.
} 
connection with his previous regular seller. Switching regular sellers leads to the endogenous dissolution of matches.

The function $m(B, S)$ determines the flow of new meetings; $m(B, S)$ has constant returns to scale, is increasing and concave in both arguments, and satisfies $m(0, S)=m(B, 0)=0$

and the Inada conditions. Let $\theta=\frac{B}{S}$ be the buyer-seller ratio, and let $\alpha_{B}(\theta)=\frac{m(B, S)}{B}$ be the rate at which a buyer meets with a new seller and $\alpha_{S}(\theta)=\frac{m(B, S)}{S}$ the rate at which a seller meets with a new buyer. Our assumptions imply that $\alpha_{B}(\cdot)$ is strictly decreasing and $\alpha_{S}(\cdot)$ is strictly increasing in $\theta$.

2. At a repeat meeting, a matched buyer meets with his regular seller. The main assumption about sellers' behavior is that, once they decide on the quality level that they offer, they commit to their decision forever. ${ }^{16}$ That is, a seller supplies the same quality at all times, and, as a result, the buyer knows the quality that he will receive from a particular seller once he has sampled his product. A match dissolves at an exogenous rate $\delta$, in addition to endogenous dissolution.

The flow of repeat meetings equals $\gamma$, which is the rate at which a matched buyer contacts his regular seller.

Buyers decide whether to participate in the market depending on their preference $z$ and, if so, their reservation quality when unmatched for matching with a new seller (the reservation quality of matched buyers for switching regular sellers is the quality that they receive from their current regular seller). Thus, buyers' actions determine the measure of participating buyers $B$ and the distribution $H(\cdot)$ of their reservation qualities. Sellers decide whether to participate in the market and, if so, what quality to offer in order to maximize their steady-state profits, depending on their cost $c$. Thus, sellers' actions determine the measure of participating sellers $S$ and the distribution $F(\cdot)$ of offered qualities.

We are ready to define the equilibrium.

Definition 1 An equilibrium consists of the actions of buyers $\{B, H(\cdot)\}$ and the actions of sellers $\{S, F(\cdot)\}$ such that entry, reservation qualities and offered qualities are chosen optimally and the market is in steady state.

\subsection{The Buyers}

We derive buyers' optimal actions, taking as given the distribution of offered qualities $F(\cdot)$ and the number of participating sellers $S$.

\footnotetext{
${ }^{16}$ See Section 3 for a discussion of the difficulties of identifying a model with alternative assumptions.
} 
An unmatched buyer with preference $z$ meets a new seller at rate $\alpha_{B}(\theta)$. At the meeting, he pays price $p$ and receives quality $x$, which, from an ex-ante point of view, is a random drawn from $F(\cdot)$. If the quality level $x$ exceeds his reservation $R_{z}$, the buyer matches with the seller, thereby obtaining capital gain $V_{z}(x)-\bar{V}_{z}$, where $V_{z}(x)$ is the value of being matched with a seller offering $x$, and $\bar{V}_{z}$ is the value of being unmatched. The value of an unmatched type- $z$ buyer satisfies:

$$
r \bar{V}_{z}=\alpha_{B}(\theta)\left(z \int_{0}^{\bar{q}} x d F(x)+\int_{R_{z}}^{\bar{q}}\left(V_{z}(x)-\bar{V}_{z}\right) d F(x)-p\right)
$$

A type- $z$ buyer participates in the market if and only if $r \bar{V}_{z} \geq K_{B}$.

A buyer with preference $z$ who is matched with a seller offering quality $q$, may experience three events: (1) at rate $\gamma$, he meets his regular seller, pays $p$ and receives $q$; $(2)$ at rate $\delta$, an exogenous shock destroys his match, and the buyer becomes unmatched, obtaining a (negative) capital gain $\bar{V}_{z}-V_{z}(q)$; and $(3)$ at rate $\alpha_{B}(\theta)$, he meets a new seller, pays $p$ and receives quality $x$, drawn from the quality distribution $F(\cdot)$. If the new seller's quality exceeds $q$, then the buyer matches with the new seller and leaves his current seller, thereby obtaining capital gain $V_{z}(x)-V_{z}(q)$. The value of a type- $z$ buyer matched with a seller offering $q$ satisfies:

$$
r V_{z}(q)=\gamma(z q-p)+\alpha_{B}(\theta)\left(z \int_{0}^{\bar{q}} x d F(x)+\int_{q}^{\bar{q}}\left(V_{z}(x)-V_{z}(q)\right) d F(x)-p\right)+\delta\left(\bar{V}_{z}-V_{z}(q)\right)
$$

The Proposition characterizes buyers' decision to participate and their reservation qualities.

Proposition 2 Given $F(\cdot)$ and $\theta$ :

1. The value of participating in the market for an unmatched type-z buyer satisfies:

$$
r \bar{V}_{z}=\alpha_{B}(\theta)\left(z \int_{0}^{\bar{q}} x d F(x)+z \int_{\frac{p}{z}}^{\bar{q}} \frac{\gamma(1-F(x))}{r+\delta+\alpha_{B}(\theta)(1-F(x))} d x-p\right)
$$

2. The optimal reservation quality for an unmatched type-z buyer is:

$$
R_{z}=\frac{p}{z}
$$

3. There exists $\hat{z}(F, \theta)$ such that a type-z buyer participates in the market if and only if $z \geq$ $\hat{z}(F, \theta)$.

Proof. Proofs of all Propositions of Section 4 are in Appendix A.

Notice that the reservation quality is decreasing in buyers' marginal utility. For a given $q$, a buyer's utility from consuming is increasing in $z$ and, thus, so is his willingness to match with the 
seller who offers $q$. Therefore, while $\gamma$ is homogeneous across buyers, buyers with higher taste for drugs endogenously meet their regular suppliers more frequently than buyers with a lower taste. Furthermore, $R_{z}$ does not depend on the distribution of offered qualities, $F(\cdot)$, because the arrival rate of new sellers is the same when matched and unmatched.

We now aggregate buyers' decisions, thereby obtaining the measure of participating buyers and the buyer-seller ratio $\theta$. We have the following characterization of the market:

Proposition 3 Given $F(\cdot)$ and $S$ :

1. If $p \geq \bar{z} \int_{0}^{\bar{q}} x d F(x)$, then there is no buyer entry: $B=0$.

2. If $p<\bar{z} \int_{0}^{\bar{q}} x d F(x)$, then there is a unique marginal buyer type $z^{*} \leq \bar{z}$ such that buyers with $z \geq z^{*}$ participate in the market and buyers with $z<z^{*}$ do not.

3. The marginal buyer type is given by the solution to:

$$
\alpha_{B}\left(\frac{\bar{B}\left(1-\bar{M}\left(z^{*}\right)\right)}{S}\right)\left(z^{*} \int_{0}^{\bar{q}} x d F(x)+z^{*} \int_{p / z^{*}}^{\bar{q}} \frac{\gamma(1-F(x))}{r+\delta+\alpha_{B}\left(\frac{\bar{B}\left(1-\bar{M}\left(z^{*}\right)\right)}{S}\right)(1-F(x))} d x-p\right)=K_{B} .
$$

The Proposition's results are intuitive. If the average quality in the market is low enough that the most eager buyer receives no surplus from a purchase, then no buyers enter the market. ${ }^{17}$ Otherwise, there is a unique marginal type such that buyers enter only if their marginal utility of consuming drugs is higher than the marginal type's.

We now describe the distribution of buyer types and reservation qualities. Let $z(R)$ denote the buyer type whose reservation quality equals $R$. Rearranging equation (4), we have:

$$
z(R)=\frac{p}{R}
$$

Moreover, note that $R_{z(R)}=R$ and $z \leq z(R) \Leftrightarrow R_{z} \geq R$. Given $z^{*}$, the equilibrium distribution of reservation qualities mirrors the distribution of marginal utilities. The corollary summarizes the results.

Corollary 4 The marginal buyer type $z^{*}$ completely characterizes buyers' behavior.

1. The measure of buyers in the market is:

$$
B=\bar{B}\left(1-\bar{M}\left(z^{*}\right)\right) .
$$

\footnotetext{
${ }^{17}$ Though intuitive, this is not immediate because the option value of climbing the quality ladder needs to be taken into account.
} 
2. The distribution of reservation qualities in the market retains the log-concavity of $\bar{M}(\cdot)$ and satisfies:

$$
H(R)= \begin{cases}0 & \text { if } R \leq \underline{R}, \\ \frac{1-\bar{M}\left(\frac{p}{R}\right)}{1-\bar{M}\left(z^{*}\right)} & \text { if } R \in[\underline{R}, \bar{R}], \\ 1 & \text { if } R \geq \bar{R},\end{cases}
$$

where $\underline{R}=R_{\bar{z}}=\frac{p}{z}$ and $\bar{R}=R_{z^{*}}=\frac{p}{z^{*}}$.

\subsection{The Sellers}

We derive sellers' optimal actions, taking as given the marginal buyer type $z^{*}$, which determines the measure $B$ of buyers who participate and the distribution of reservation qualities $H(\cdot)$.

Steady-state profits of a type- $c$ seller offering quality $q$ are:

$$
\pi_{c}(q)=t(q)(p-c q)
$$

where $t(q)$ denotes the steady-state flow of transactions when offering $q$, and $p-c q$ is the margin per transaction. A type- $c$ seller enters the market if and only if $\pi_{c}\left(q^{*}(c)\right) \geq K_{S}$, where $q^{*}(c)$ denotes the quality level that maximizes his steady-state profits.

An individual seller's transactions come from two sources: new buyers, who purchase from that seller for the first time, and repeat buyers, who purchased from that seller in the past and decided to match with him. The flow of transactions is $t(q)=t_{N}+t_{R}(q)$, where $t_{N}$ represents sales to new buyers, and $t_{R}(q)$ represents sales to the seller's regular buyers. Steady-state profits, therefore, equal:

$$
\pi_{c}(q)=\left(t_{N}+t_{R}(q)\right)(p-c q)
$$

The flow of new transactions equals the rate at which new buyers contact an individual seller, which does not depend on the quality offered:

$$
t_{N}=\alpha_{S}(\theta)=\theta \alpha_{B}(\theta)
$$

The flow of repeat transactions to a seller who offers quality $q$ depends on the number of regular buyers, denoted by $l(q)$, and the rate $\gamma$ at which these buyers contact their regular seller:

$$
t_{R}(q)=\gamma l(q)
$$

Unlike new transactions, the flow of repeat transactions depends on the seller's offered quality. A 
seller who offers quality $q$ gains regular customers when he meets with unmatched buyers whose reservation is below $q$ and with matched buyers whose regular seller offers less than $q$. Similarly, he loses his regular customers when they meet with a seller offering quality greater than $q$ and when the match exogenously dissolves, which happens at rate $\delta$.

We characterize sellers' optimal actions in three steps. First, we derive some necessary conditions for the distribution of offered qualities. Second, we derive an individual seller's profits. Third, we characterize the full distribution of offered qualities and sellers' entry decisions.

In equilibrium, the quality distribution displays the following features:

Lemma 5 In equilibrium, the quality distribution $F(\cdot)$ is continuous on $[0, \bar{q}]$ and has support on a subset of $\{0\} \cup[\underline{q}, \bar{q}]$; the support is connected on $[\underline{q}, \bar{q}]$ for some $\underline{q} \in[\underline{R}, \bar{R}]$.

We can derive the steady-state profits of a type- $c$ seller, given the actions of buyers and of other sellers.

Proposition 6 Given $H(\cdot), \theta$ and $F(\cdot)$, the steady-state profits of a type-c seller who offers quality q are:

$$
\pi_{c}(q)= \begin{cases}\alpha_{B}(\theta) \theta\left(1+\frac{\gamma \delta H(q)}{\left(\delta+\alpha_{B}(\theta)(1-F(q))\right)^{2}}\right)(p-c q) & \text { if } q \geq \underline{R} \\ \alpha_{B}(\theta) \theta p & \text { if } q<\underline{R} .\end{cases}
$$

We can then characterize sellers' optimal decisions, given the measure of buyers $B$ and their reservation distribution $H(\cdot)$.

\section{Proposition 7 Given $B$ and $H(\cdot)$ :}

1. A positive measure of sellers offer positive quality in the market. There is a unique marginal seller type $c^{*}$ such that sellers with $c \leq c^{*}$ enter the market and offer quality $q^{*}(c)>0$, which is strictly decreasing in $c$.

2. Measure $S_{0}$ of sellers offer zero quality. $S_{0}>0$ if and only if the measure of potential sellers is below some threshold.

The subsequent quantitative analysis will focus on the case in which cheating occurs in equilibrium $\left(S_{0}>0\right)$, as this is the empirically relevant case.

Proposition 7 illustrates the importance of imperfect observability of quality and moral hazard for market outcomes: sellers with a high cost of providing drugs participate in the market but specialize in cheating their buyers - i.e., they offer zero quality - and still retain a positive flow of sales. In a market with perfect observability of quality, such sellers would not be in the market. 
Corollary 8 Seller behavior is summarized as follows:

1. The measure of sellers in the market is $S=\bar{S} \bar{D}\left(c^{*}\right)+S_{0}$.

2. The distribution of sellers who offer positive quality is $D(c)=\frac{\bar{D}(c)}{\bar{D}\left(c^{*}\right)}$ for $c \leq c^{*}$.

3. The quality distribution is $F(q)=1-(1-F(0)) D\left(q^{*-1}(q)\right)$ for $q>0$ and $F(0)=\frac{S_{0}}{S}$.

\subsection{Equilibrium}

The equilibrium is a fixed point on the marginal buyer type. Given $z^{*}$, the measure of participating buyers $B$ and the distribution of their reservations $H(\cdot)$ are uniquely determined (Corollary 4). This, in turn, determines the marginal seller type $c^{*}$, the measure of sellers who enter the market $S$, (Proposition 7 ) and the quality distribution $F(\cdot)$ (Corollary 8 ). Finally, $F(\cdot)$ and $S$ determine the marginal buyer type (Proposition 3, equation (5)). The marginal type is defined on a closed and bounded set $[0, \bar{z}]$, and Proposition 9 follows.

Proposition 9 An equilibrium exists.

\section{Empirical Analysis}

The model does not admit an analytic solution for all endogenous outcomes. Hence, we choose the parameters that best match moments of the data with the corresponding moments computed from the model's numerical solution. We then study the quantitative implications of the model evaluated at the estimated parameters.

\subsection{Parametric Assumptions}

We estimate the model using the data described in Section 3, assuming that they are generated from the model's steady state. We further assume that the empirical quality distribution obtained from STRIDE corresponds to the distribution $F(\cdot)$ that first-time buyers face. We set the unit of time to be one month, as this is the period over which we observe consumption frequencies in ADAM.

Unfortunately, the data lack some detailed information to identify all parameters. Therefore, we fix some values. Specifically, the discount rate $r$ is traditionally difficult to identify, and we set it to $r=0.01$. Moreover, since we use the normalized variable PURE Grams PER $\$ 100$, we set the price equal to $p=\$ 100$. Furthermore, we set sellers' monthly flow cost $K_{S}$ to be $\$ 1,500$, which is broadly in line with the bottom of the distribution of drug dealers' earnings reported by Levitt and Venkatesh (2000). 
We further make parametric assumptions about the distributions of buyers' and sellers' heterogeneity. We borrow these assumptions from the papers that structurally estimate search models of the labor market. Notably, the shape of the drug quality distribution displayed in Figure 1 resembles the shape of the wage distribution-i.e., it is approximately lognormal with a long tail. However, there are two important differences: 1) the quality distribution displays the spike at zero - thus our focus on sellers' moral hazard to explain it; and 2) the quality distribution is a distribution of offers $(F(\cdot)$ in our notation), whereas the wage distribution is a distribution of accepted offers $(G(\cdot)$ in our notation). Some papers that structurally estimate search models of the labor market based on Burdett and Mortensen (1998), such as Bontemps et al. (1999), use a lognormal distribution for workers' productivity and a Pareto distribution for firm productivity. Hence, given the similarities in modeling frameworks and empirical targets, we choose a lognormal distribution for buyers and a Pareto distribution for sellers, as well.

More precisely, we assume that the distribution $\bar{M}(\cdot)$ of buyers' taste for crack cocaine $z$ is a mixture distribution: a fraction $\lambda$ has taste $z=0$, so they will never enter the market; a fraction $1-\lambda$ has taste $z$ that follows a lognormal distribution with unknown parameters $\mu_{z}$ and $\sigma_{z}$ and, for consistency with our theoretical model, is truncated above at the upper bound $\bar{z}$ that satisfies $\log (\bar{z})=\mu_{z}+3 \sigma_{z}$. Hence, the maximum number of active buyers is $(1-\lambda) \bar{B}$. ${ }^{18}$ Since the reservation quality of a buyer with type $z>0$ is $R_{z}=\frac{p}{z}$, it follows that the distribution $H(\cdot)$ of reservation qualities is also lognormal with parameters $\mu_{R}=\log p-\mu_{z}$ and $\sigma_{R}=\sigma_{z}$.

Moreover, we assume that the distribution of the inverse of sellers' costs $1 / c$ is a Pareto distribution with lower bound $\frac{1}{\bar{c}}$ and shape parameter $\xi \geq 1$. This implies that the distribution of $\operatorname{costs} c$ is:

$$
\bar{D}(c)=\left(\frac{c}{\bar{c}}\right)^{\xi}, c \in[0, \bar{c}]
$$

and, thus, the truncated distribution of active sellers' costs equals

$$
D(c)=\left(\frac{c}{c^{*}}\right)^{\xi}, c \in\left[0, c^{*}\right],
$$

where $c^{*}$ is the cost of the marginal active seller that offers the lowest quality level $\underline{q}$. The shape parameter $\xi$ captures the dispersion of costs. If $\xi=1$, the cost distribution is uniform. As $\xi$ increases, the relative number of high-cost sellers increases, and the cost distribution is more concentrated at these higher cost levels. As $\xi$ goes to infinity, the cost distribution becomes degenerate at the upper bound. ${ }^{19}$

\footnotetext{
${ }^{18}$ An alternative rationalization of the total size of the market is that buyers have heterogeneous flow costs $K_{B}$, and buyers with high $K_{B}$ do not enter the market. However, we should point out that buyers' flow cost $K_{B}$ affects only their entry decision, whereas the value of $z$ additionally affects their consumption. Hence, the heterogeneity of buyers' consumption observed in the data is directly informative about (i.e., identifies) the heterogeneity of preferences $z$.

${ }^{19}$ In the absence of the measurement error $\epsilon$ specified below, we could non-parametrically estimate the distri-
} 
We further assume that drug qualities $q$ and the number of purchases are measured with errors. Specifically, we assume that the reported qualities $\hat{q}$ and the "true" qualities $q$ are related as: $\hat{q}=q \epsilon$, where $\epsilon$ is a measurement error, drawn from a lognormal distribution with parameters $\left(\mu_{\epsilon}, \sigma_{\epsilon}\right)$, and with mean to equal 1 -i.e., measurements are unbiased; hence, the parameters $\left(\mu_{\epsilon}, \sigma_{\epsilon}\right)$, satisfy $\mu_{\epsilon}=-0.5 \sigma_{\epsilon}^{2}$. Similarly, we assume that the reported number of purchases depends on the product of the "true" number of purchases and a measurement error $\nu$, which is independent of $\epsilon$, and drawn from a lognormal distribution with parameters $\left(\mu_{\nu}, \sigma_{\nu}\right)$, and with mean to equal 1: hence, $\mu_{\nu}=-0.5 \sigma_{\nu}^{2}$. Moreover, to preserve the discreteness of the reported number of purchases, we round the resulting product of the "true" number of purchases and the measurement error $\nu$ to the nearest integer.

The assumption of measurement error on wages is quite common in the literature that structurally estimates search models of the labor market. In our application, it is plausible that drug quality is measured with error in STRIDE. Measurement error could also account for some unobserved seller behavior that the model does not consider (i.e., price discrimination), thereby allowing us to fit the quality distribution better. For example, as Lemma 5 highlights, the model implies a gap in the quality distribution between the complete rip-offs $q^{*}=0$ and the minimum positive quality $\underline{q}$. Figure 1 shows that the empirical distribution displays this qualitative feature, and the measurement $\epsilon$ allows it to more precisely match its magnitude; the figure also shows that the quality distribution displays a long right tail, and the measurement $\epsilon$ allows the model to capture some of these high-quality transactions. Similarly, the measurement error on the number of purchases can account for the fact that respondents to the ADAM survey may have imperfect (although not systematically biased) recall of their recent purchases; in addition, it allows us to better fit moments of the distribution of the number of purchases.

Finally, we explicitly model the selection into the ADAM sample. Specifically, we assume that an individual who does not consume drugs is in ADAM if $\eta \geq 0$, irrespective of his type $z$, whereas an individual of type $z$ who consumes drugs is in $\operatorname{ADAM}$ if $\log (z)+\eta \geq 0$, where $\eta$ is a random variable that is independent of $z$ and is distributed according to a normal distribution with mean $\mu_{\eta}$ and standard deviation $\sigma_{\eta}$. Hence, this selection process highlights that users are more likely to be in ADAM than non-users, and that buyers with higher preferences for drugs (and, thus, greater drug consumption) are more likely to be in the ADAM dataset than buyers with lower preferences (and, thus, lower consumption). Appendix B reports the details of the derivation of the density of drug users' preferences $z$ in ADAM; we will use this density to compute simulated moments that we match to their empirical counterparts.

bution $D(c)$ from the empirical distribution of $q$ for $q>0$, as $D(c)=\frac{1-F(q)}{1-F(0)}$. However, a parametric distribution for $D(c)$ facilitates the counterfactual out-of-sample analyses of Section 5.4. 


\subsection{Estimation and Identification}

We estimate the vector $\psi=\left\{\alpha_{B}, \gamma, \delta, K_{B}, \mu_{z}, \sigma_{z}, c^{*}, \xi, \sigma_{\epsilon}, \sigma_{\nu}, \mu_{\eta}, \sigma_{\eta}, \lambda\right\}$ using a minimumdistance estimator that matches key moments of the data with the corresponding moments of the model. ${ }^{20}$ More precisely, for any value of the vector $\psi$, we solve the model of Section 4 to find its equilibrium: the mass $B$ of active buyers and their distribution of reservation qualities $H(\cdot)$, and the mass $S$ of active sellers and their distribution $F(\cdot)$ of offered qualities. We then calculate two sets of moments, one that we match to a set of moments computed from the STRIDE dataset and one that we match to a set of moments computed from the ADAM dataset.

The first set $\mathbf{m}_{1}(\psi)$ is composed of these moments of the offered quality distribution $F(\cdot)$ :

1. The fraction of rip-offs $q=\hat{q}=0$.

2. The mean of quality for $\hat{q}>0$.

3. The standard deviation of quality for $\hat{q}>0$.

4. The median of quality for $\hat{q}>0$.

5. The skewness of quality for $\hat{q}>0$.

6. The kurtosis of quality for $\hat{q}>0$.

Moreover, at each value of the parameters, we simulate buyer-seller meetings and consumption patterns (i.e., the $\alpha_{B}, \delta$ and $\gamma$ shocks), using the distributions of preferences $z$ and buyer-seller matches that take into account the selection into ADAM (see Appendix B). We then compute the second set $\mathbf{m}_{2}(\psi)$ composed of these moments:

1. The fraction of individuals who purchased crack cocaine in the last 30 days.

2. The fraction of users who made their last purchase from their regular dealer, among those who purchased crack cocaine in the last 30 days (in the simulation, a purchase from a regular dealer is defined as a purchase from the same seller as the previous purchase).

3. The average number of purchases of those who purchased crack cocaine in the last 30 days and made their last purchase from their regular dealer.

4. The average number of purchases of those who purchased crack cocaine in the last 30 days and did not make their last purchase from their regular dealer.

\footnotetext{
${ }^{20}$ While $\alpha_{B}$ is the endogenous rate of new meetings and $c^{*}$ is the cost of the marginal seller that (endogenously) offers the lowest quality level $\underline{q}$, we can infer them from the data, and this inference, along with additional assumptions, allows us to perform counterfactual analyses, as we explain in Section 5.4.
} 
5. The standard deviation of the number of purchases of those who purchased crack cocaine in the last 30 days and made their last purchase from their regular dealer.

6. The standard deviation of the number of purchases of those who purchased crack cocaine in the last 30 days and did not make their last purchase from their regular dealer.

7. The median of the number of purchases of those who purchased crack cocaine in the last 30 days and made their last purchase from their regular dealer.

8. The median of the number of purchases of those who purchased crack cocaine in the last 30 days and did not make their last purchase from their regular dealer.

We add to this set two moments calculated from our auxiliary data that identify the parameters that determine the selection into the ADAM sample and the fraction $\lambda$ of the population who has no taste for crack cocaine.

9. The fraction of individuals aged 15 and older who report consuming crack cocaine in the 2001-2003 NSDUH. Equation B2 in Appendix B derives the analytical formula for this fraction.

10. The fraction of individuals arrested. Equation B1 in Appendix B derives the analytical formula for this fraction.

The minimum-distance estimator chooses the parameter vector $\psi$ that minimizes the criterion function

$$
\left(\mathbf{m}(\psi)-m_{S}\right)^{\prime} \Omega\left(\mathbf{m}(\psi)-m_{S}\right)
$$

where $\mathbf{m}(\psi)=\left[\begin{array}{l}\mathbf{m}_{1}(\psi) \\ \mathbf{m}_{2}(\psi)\end{array}\right]$ is the vector of stacked moments computed from the model evaluated at $\psi$, and $m_{S}$ is the vector of corresponding sample moments. $\Omega$ is a symmetric, positive-definite matrix; in practice, we use a diagonal matrix whose elements are those on the main diagonal of the inverse of the matrix $E\left(m_{S}^{\prime} m_{S}\right)$.

The identification of the model is similar to that of structural search models of the labor market that follow the framework of Burdett and Mortensen (1998); see, for example, Bontemps et al. (1999). Specifically, although the model is highly nonlinear, so that (almost) all parameters affect all outcomes, the identification of some parameters relies on some key moments in the data.

The moments of the quality distribution identify the parameters of the distribution $D(\cdot)$ of sellers' heterogeneity and of the distribution of the measurement error $\epsilon$ on drug quality, and they contribute to the identification of the parameters of the distribution $M(\cdot)$ of buyers' heterogeneity. More precisely, in the absence of measurement error on $q$, the empirical distribution of $q$ for 
$q>0$ non-parametrically identifies the distribution $D(c)$ since Corollary 8 shows that $F(q)=$ $1-(1-F(0)) D\left(q^{*-1}(q)\right)$ for $q>0$. Moreover, as in structural search models of the labor market, the data sometimes display events that should not occur according to the model, and these "zero-probability events" identify the parameters of the distributions of the measurement errors. In search models of the labor markets, these events include job-to-job transitions that feature a wage decrease, for example; instead, our model implies a gap in the quality distribution between $q=0$ and $\underline{q}$ that is larger than that observed in the data-i.e., no seller offers a small positive quality, as this quality is strictly more expensive than zero quality and does not induce buyers to purchase again from the seller offering it - and this gap identifies the parameter $\sigma_{\epsilon}$ of the distribution of $\epsilon$.

The moments of buyers' consumptions identify the meeting rates $\alpha_{B}$ and $\gamma$, the destruction rate $\delta$, the parameter $\sigma_{\nu}$ of the distribution of the measurement error on the number of purchases, and they contribute to the identification of the parameters of the distribution $M(\cdot)$ of buyers' heterogeneity. Specifically, the difference between the average number of purchases of those who made their last purchase from their regular dealer and of those who did not identify the parameter $\gamma$, whereas the fraction of users who made their last purchase for their regular dealer and the unconditional average number of purchases jointly identify the meeting rates $\alpha_{B}$ and the destruction rate $\delta$. The moments of the distribution of the number of purchases contribute to the identification of the parameters of the distribution $M(\cdot)$ of buyers' heterogeneity and identify the parameter $\sigma_{\nu}$ of the distribution of the measurement error $\nu$ on the number of purchases. In particular, the model without this measurement error cannot fully account for the observed heterogeneity in (i.e., the standard deviation of) the number of purchases across individuals, and the measurement error $\nu$ allows the empirical model to match this feature of the data; thus, this difference between the theory and the data identifies the parameter $\sigma_{\nu}$ of the distribution of $\nu{ }^{21}$

Finally, the fraction of individuals who purchased crack cocaine in the last 30 days in the ADAM data, the fraction of individuals consuming crack cocaine in the NSDUH, and the arrest rate identify the fraction $\lambda$ of individuals who have taste $z=0$ for drugs, as well as the parameters of the distribution of the unobservable $\eta$ that contributes to the selection into the ADAM sample. ${ }^{22}$ Using the estimated distribution of buyers' preferences, we then recover buyers' cost

\footnotetext{
${ }^{21}$ If addicted individuals are those whose number of purchases in ADAM exceeds a threshold, we could understand how the presence of addicts affects our results by estimating our model on a sample without these individuals (Jacobi and Sovinsky, 2016). As a result of this removal, the average and the standard deviation of the number of purchases will be lower than those reported in Table 1. Hence, the parameter $\gamma$ and the standard deviation $\sigma_{\nu}$ of the measurement error $\nu$ will likely be lower than those reported in Table 2. However, we believe that the key implications of the model will be unaffected, as the model and the counterfactuals do not depend on the specific values of $\gamma$ and $\sigma_{\nu}$.

${ }^{22}$ Since we calculate the arrest rate using aggregate data, this moment has no sampling variability. Hence, the confidence intervals of the estimates of $\lambda, \mu_{\eta}$ and $\sigma_{\eta}$ do not depend on the sampling variability of the arrest rate, but exclusively on the sampling variability of the moments that identify the distribution of the taste $z$. See Imbens and Lancaster (1994) on combining moments from different samples.
} 
TABLE 2: Estimates

\begin{tabular}{cccc}
\hline \hline$\alpha_{B}$ & 1.267 & $\lambda$ & 0.982 \\
& {$[1.217,1.267]$} & & {$[0.980,0.983]$} \\
$\gamma$ & 19.399 & & 5.118 \\
& {$[18.868,20.658]$} & $\mu_{z}$ & {$[5.097,5.137]$} \\
$\delta$ & 0.731 & & 0.114 \\
& {$[0.700,0.734]$} & $\sigma_{z}$ & {$[0.103,0.125]$} \\
$K_{B}$ & 152.639 & & 124.368 \\
& {$[112.710,166.035]$} & $c^{*}$ & {$[123.597,128.681]$} \\
$\sigma_{\epsilon}$ & 0.526 & & 20.443 \\
& {$[0.457,0.572]$} & $\xi$ & {$[20.443,31.284]$} \\
$\sigma_{\eta}$ & 2.803 & & -5.237 \\
& {$[2.723,2.852]$} & $\mu_{\eta}$ & {$[-5.329,-5.103]$} \\
$\sigma_{\nu}$ & 0.522 & & \\
\hline \hline
\end{tabular}

Notes-This table reports the estimates of the parameters. 95-percent confidence intervals in brackets are obtained by bootstrapping the data using 100 replications.

$K_{B}$ from the entry condition of the marginal buyer, equation (5).

\subsubsection{Estimates and Model Fit}

Table 2 reports estimates of the parameters, along with 95-percent confidence intervals obtained by bootstrapping the data using 100 replications.

The value of $\alpha_{B}$ indicates that a buyer meets a new seller, on average, approximately every $\frac{30}{\alpha_{B}} \approx 24$ days. The value of $\gamma$ indicates that a matched buyer purchases, on average, approximately 19 times per month. However, the buyer-seller match exogenously breaks, on average, every $\frac{30}{\delta}=41$ days. ${ }^{23}$ Buyers' monthly cost $K_{B}$ is quite low, approximately $\$ 150$.

The estimates of the parameters of the distribution of buyers' heterogeneity imply that 98 percent of individuals have taste $z$ equal to zero, and, thus, the market for crack cocaine is limited in size, presumably because crack cocaine is one of the most addictive and dangerous drugs. Of those individuals with positive preferences, 82 percent are active in the market, which corresponds to buyers with taste $z \geq z^{*}=150.71$; among those active buyers, the average taste is approximately equal to 175 and the standard deviation 16 . The value of $c^{*}$ and of the shape parameter $\xi$ of sellers' cost distribution imply that the range of costs of sellers offering positive quality is $[0,124.37]$, but their average cost is 118.56 , as $\xi=20.44$ implies that almost all these sellers have costs quite close to the cost $c^{*}$ of the marginal seller that offers the lowest quality

\footnotetext{
${ }^{23}$ Reuter et al. (1990) reports evidence consistent with high turnover of buyer-seller relationships.
} 
level $\underline{q}$. The comparison between buyers' average valuation and sellers' average cost implies that the surplus of each pure gram traded equals approximately $\$ 55$ dollars. Moreover, the density of buyers' preferences evaluated at the entry threshold $z^{*}$ and the density of sellers' costs evaluated at the threshold $c^{*}$ imply that the demand for drugs is substantially less elastic than the supply.

Finally, we estimate that $\sigma_{\epsilon}$ equals 0.52 , which means that the variance of the measurement error on drug quality equals 0.32 . This value, along with those of the other estimates, implies that the model without measurement error $\epsilon$ accounts for approximately 60 percent of the dispersion of drug quality observed in the data, and that the error $\epsilon$ improves the fit, in particular by "filling the gap" between $q=0$ and $\underline{q}=0.63$, and by rationalizing the highest- $q$ transactions. ${ }^{24}$ Similarly, we estimate that $\sigma_{\nu}$ equals 0.52 , which means that the variance of the measurement error on the number of purchases equals 0.31 . The parameters imply that the model without measurement error accounts for approximately 75 percent of the dispersion of drug quality; the measurement error $\nu$ increases this dispersion, thereby improving the fit.

Table 3 presents a comparison between the empirical moments and the moments calculated from the model at the estimated parameters. Overall, the model matches the moments of the quality distribution well: most notably, it perfectly captures the fraction of rip-offs and the average quality of drugs. Similarly, the model perfectly captures the difference in consumption rates between matched and unmatched buyers, as well as the fraction of matched buyers. Moreover, the model exactly matches the fraction of individuals purchasing crack cocaine in the ADAM sample, the fraction of individuals consuming crack cocaine in the NSDUH, as well as the arrest rate, indicating that our empirical model captures the over-representation of drug users in the ADAM sample very well.

\subsection{Model Implications}

The estimated parameters reported in Table 2 imply that 15.6 percent of all sellers rip their buyers off by choosing $q=0$. For sellers with costs $0 \leq c \leq c^{*}=124.37, q^{*}(c)$ is the solution to the differential equation (A5) in Appendix A: sellers' quality choices are strictly decreasing in their costs, as Lemma 7 says.

Sellers' quality choice $q^{*}(c)$ implies that sellers' markups $\frac{p-c q^{*}(c)}{p}$ are non-monotonic in $c$, with the lowest- and highest-cost sellers charging the highest markups (equal to 1, as either $c$ or $q$ equals 0 ), and the seller with cost $c=114.92$ charging the lowest one; the average sellers' markup $\frac{\int_{0}^{\bar{c}}\left(p-c q^{*}(c)\right) d D(c)}{p}$ equals 13.43 percent. $^{25}$ On average, sellers make approximately 175 transactions

\footnotetext{
${ }^{24}$ Of course, if we estimate the model without measurement error, the estimated variance of $q$ increases.

${ }^{25}$ The STRIDE data allow us to corroborate these estimates of dealers' costs/margins. Specifically, while it is not straightforward to define the wholesale market (and know how it works; for example, we may also need to consider the role of long-term relationships in the wholesale market), we use powder cocaine as the main input of crack cocaine and assume that retail sellers buy powder cocaine in transactions of values between $\$ 200$ and $\$ 1000$,
} 
TABle 3: Model Fit

\begin{tabular}{lcc}
\hline \hline & Data & Model \\
\hline Fraction of Rip-offs $(\%)$ & 15.338 & 15.862 \\
Average Pure Grams Per $\$ 100, \hat{q}>0$ & 0.735 & 0.732 \\
St. Dev. Pure Grams Per $\$ 100, \hat{q}>0$ & 0.505 & 0.416 \\
Median Pure Grams Per $\$ 100, \hat{q}>0$ & 0.591 & 0.635 \\
Skewness Pure Grams Per $\$ 100, \hat{q}>0$ & 1.952 & 1.870 \\
Kurtosis Pure Grams Per $\$ 100, \hat{q}>0$ & 8.516 & 9.521 \\
Fraction Obtained Drug in Last 30 Days $(\%)$ & 16.900 & 16.899 \\
Fraction Last Purchased From Regular Dealer $(\%)$ & 52.481 & 52.960 \\
Average Number of Purchases, Matched Buyer & 16.331 & 16.771 \\
Average Number of Purchases, Unmatched Buyer & 11.548 & 10.756 \\
St. Dev. Number of Purchases, Matched Buyer & 11.124 & 11.477 \\
St. Dev. Number of Purchases, Unmatched Buyer & 10.419 & 10.337 \\
Median Number of Purchases, Matched Buyer & 15.000 & 14.000 \\
Median Number of Purchases, Unmatched Buyer & 7.000 & 7.000 \\
Fraction Consuming Drug in NSDUh $(\%)$ & 0.800 & 0.800 \\
Arrest Rate $(\%)$ & 3.776 & 3.775 \\
\hline \hline
\end{tabular}

Notes - This table reports the values of the empirical moments and of the simulated moments calculated at the estimated parameters reported in Table 2.

$t(q)$ per month, and the distribution of transactions $t(q)$ has a large range - the lowest-quality (i.e., highest-cost) sellers make approximately 70 monthly deals, and the highest-quality sellers make approximately 410 monthly deals - and is skewed towards sellers with fewer transactions. Sellers' profits have a large range and are highly skewed, as well: the lowest-quality seller earns $\$ 1,500$ per month; the highest-quality seller earns approximately $\$ 13,000$ per month; and the average seller earns approximately $\$ 2,300$. The shape of the distribution of profits matches the evidence reported by Reuter et al. (1990) and Levitt and Venkatesh (2000) reasonably well.

The distribution $G(q)$ of qualities from which matched buyers consume differs substantially from the distribution $F(q)$ of qualities from which unmatched buyers consume. The distribution $F(q)$ displays the key features of the distribution of qualities characterized in Lemma 5 , most notably the mass point at zero quality. Of course, no matched buyer purchases zero quality from

which implies an approximate average value of $\$ 500$. Assuming that transactions in STRIDE are representative of this wholesale market, we obtain an average cost of a pure gram of powder cocaine of $\$ 111.96$ and, thus, an average margin of approximately 12 percent. Hence, these values are quite close to our estimated average costs and margins. 
his regular dealer; hence, while approximately 15 percent of sellers offer zero-quality drugs, the fraction of all transactions that feature zero quality equals $\frac{B \alpha_{B} * F(0)}{\left(B \alpha_{B}+B-\bar{n}\right) \gamma}=1.57$ percent only, as all $B$ active buyers consume at rate $\alpha_{B}$, and $(B-\bar{n})$ matched buyers additionally consume drugs with strictly positive quality at rate $\gamma$, whereas only the share $F(0)$ of the former transactions features zero quality Moreover, as buyers move up over time in the offered quality distribution by switching to sellers that offer higher-quality drugs, they are more likely to be matched to higherquality sellers. Hence, the cumulative $G(q)$ first-order stochastically dominates the cumulative $F(q)$. Matched buyers consume drugs that have an average quality of $\int_{\underline{q}}^{\bar{q}} q g(q) d q=0.76$, whereas unmatched buyers consume drugs that have an average quality of $\int_{0}^{\bar{q}} q f(q) d q=0.62$, indicating that buyers' switching behavior and buyer-seller relationships allow regular buyers to consume drugs that are, on average, 23-percent better than the drugs that first-time buyers consume.

\subsection{Counterfactual Analyses}

In this Section, we use our model to quantitatively analyze two key features of illegal markets: 1) the effect of imperfect observability and moral hazard on market outcomes; and 2) the effect of changing penalties on market outcomes.

Both analyses are out-of-sample and, thus, require that we specify the measure $\bar{B}$ of potential buyers and the functional form of the matching function $m(B, S)$ that determines the aggregate number of new meetings between $B$ active buyers and $S$ active sellers. We can further determine the number of active sellers in each counterfactual from sellers' free entry conditions-i.e., equation (C2) in Online Appendix C in the first counterfactual on observability, and equation (A2) in Appendix A in the second counterfactual on penalties, respectively. ${ }^{26}$

We set the measure $\bar{B}$ of potential buyers to 228 million, which, as we mentioned when reporting the fraction of individuals arrested, is the U.S. population over 15 years of age reported in July 2002 (the midpoint of our sample period) by the U.S. Census.

We further assume a Cobb-Douglas functional form for the matching function:

$$
m(B, S)=\omega B^{1 / 2} S^{1 / 2}
$$

where $\omega$ is the efficiency of the matching function. With our estimated parameters, we can calculate $\omega$ as

$$
\omega=\frac{m(B, S)}{B^{1 / 2} S^{1 / 2}}=\frac{\alpha_{B}(\theta) B}{B^{1 / 2} S^{1 / 2}}
$$

where we estimate $\alpha_{B}$ using the ADAM data, we obtain $B$ from the fraction of the population consuming crack cocaine, and we infer $S$ from sellers' free-entry condition. Online Appendix D

\footnotetext{
${ }^{26}$ Thus, we do not need to specify the measure $\bar{S}$ of potential sellers, as sellers' free entry condition determines the number of active sellers in each counterfactual case.
} 
reports on the results obtained using alternative matching functions, confirming the robustness of the results that we report in this Section.

In the following counterfactual analyses, we report quantitative results for the population (i.e., without sample selection) as ratios of the corresponding values in the baseline case (i.e., a ratio larger than one implies an increase relative to the baseline case), without measurement errors on drug quality and drug purchases.

\subsubsection{The Role of Sellers' Moral Hazard}

In order to understand the quantitative effect of moral hazard on market outcomes, we modify the baseline model by letting buyers observe quality before making a purchase and compute the new equilibrium. This counterfactual highlights how the observability of $q$ affects the interaction between buyers and sellers and, thus, the equilibrium distribution of quality $q$ and agents' participation in the market. Online Appendix $\mathrm{C}$ reports the full derivation of the equilibrium.

When quality is observable, after observing $q$ in a new meeting, a buyer has two decisions to make: whether to consume and whether to match. Regardless of whether or not the buyer is currently matched, he makes a purchase when the instantaneous payoff $z q-p$ is positive. The decision to match is similar to that in the baseline model: if matched, the buyer chooses between matching with the new seller or returning to his regular seller, after potentially taking advantage of the consumption opportunity; if unmatched, the buyer chooses between matching with the new seller and remaining unmatched, as consuming and remaining unmatched is not optimal. Hence, the value functions of a type- $z$ buyer satisfy:

$$
\begin{aligned}
r \bar{V}_{z} & =\alpha_{B}(\theta) \int_{0}^{\bar{q}} \max \left[z x-p+\max \left[V_{z}(x)-\bar{V}_{z}, 0\right], 0\right] d F(x) \\
r V_{z}(q) & =\gamma(z q-p)+\alpha_{B}(\theta) \int_{0}^{\bar{q}} \max \left[z x-p+\max \left[V_{z}(x)-V_{z}(q), 0\right], 0\right] d F(x)+\delta\left(\bar{V}_{z}-V_{z}(q)\right) .
\end{aligned}
$$

Simple calculations yield that the reservation quality for consuming is the same as the reservation quality for matching (see Online Appendix C for details).

Quality observability affects sellers' payoffs because new buyers make a purchase only if their instantaneous payoff is positive. Therefore, the flow of transactions with new buyers of a seller offering quality $q$ equals the meeting rate with new buyers $\alpha_{S}(\theta)$ times the probability that the buyer's reservation is below $q, H(q)$ :

$$
t_{N}(q)=\alpha_{S}(\theta) H(q)
$$

The flow of sellers' transactions with regular buyers $t_{R}(q)$ is determined in an equivalent way to 
the baseline case (see Online Appendix C for details). Thus, steady-state profits are:

$$
\pi_{c}(q)=(p-c q)\left(t_{N}(q)+t_{R}(q)\right)
$$

Sellers' incentives differ from those in the baseline case and, thus, their choices do as well. Specifically, offering zero quality yields negative instantaneous payoff to all buyers and, thus, sellers no longer offer complete rip-offs. Therefore, the distribution of offered quality does not feature a mass point at zero. More generally, the flow of new customers depends on the quality offered since buyers with low levels of $z$ might choose not to purchase from low- $q$ sellers.

As in the baseline model, buyers and sellers choose whether to participate in the market, which now has different payoffs due to observable quality. Therefore, buyers' and sellers' endogenous entry thresholds change relative to the baseline case-i.e., they equal $z^{* *}$ and $c^{* *}$, characterized in equations (C1) and (C2), respectively, in Online Appendix C-thereby determining a different number of active buyers and active sellers relative to the unobservable quality case of Section 4 (see Corollaries 11 and 16).

We quantitatively assess the effect of eliminating moral hazard by computing the model's steady state with observable quality at the estimated parameters. We consider two separate cases: 1) a partial-equilibrium case in which the measures of participating buyers and sellers are unchanged relative to the baseline case, but they make optimal decisions in the new information environment; and 2) a general-equilibrium case in which, in addition to the partial-equilibrium optimizations, buyers and sellers also make optimal entry decisions. We believe that the partialequilibrium case is useful for focusing exclusively on the effects of moral hazard on the distribution of drug quality. The general-equilibrium case is useful because it further illustrates how moral hazard (or the lack thereof) affects agents' incentives to participate in the market.

Table 4 reports market outcomes for the counterfactuals of observable quality for the partialequilibrium case and the general-equilibrium case. Overall, market outcomes differ substantially when buyers observe drug purity and when they do not. Moreover, the quantitative effects are very similar in the partial- and general-equilibrium cases. Specifically, the partial-equilibrium case highlights that, relative to the baseline case, the average offered quality increases by approximately 20 percent, and the standard deviation of quality decreases by approximately 80 percent. Moreover, zero-purity drugs disappear from the market when quality is observable, as we discussed above. Overall, this counterfactual indicates that unobservable quality, rather than search frictions, is the main determinant of the observed dispersion of quality. Finally, a larger fraction of buyers are matched to a regular seller, thereby increasing their number of purchases and consumption by approximately five and eight percent, respectively.

The general-equilibrium case highlights additional effects relative to the partial-equilibrium case. First, buyers receive higher quality than in the baseline case, thereby increasing buyers' 
TABLE 4: Observable Quality

\begin{tabular}{|c|c|c|c|}
\hline & BASELINE & $\begin{array}{l}\text { OBSERVABLE } q \\
\text { PARTIAL EQ. }\end{array}$ & $\begin{array}{l}\text { OBSERVABLE } q \text {, } \\
\text { GENERAL EQ. }\end{array}$ \\
\hline \multirow{2}{*}{ FRACTION OF RIP-OFFS (\%) } & 15.862 & 0.000 & 0.000 \\
\hline & {$[13.646 ; 16.812]$} & {$[0.000 ; 0.000]$} & {$[0.000 ; 0.000]$} \\
\hline \multirow{2}{*}{ Average Pure Grams Per $\$ 100$} & 0.616 & 1.204 & 1.218 \\
\hline & {$[0.597 ; 0.636]$} & {$[1.160 ; 1.261]$} & {$[1.166 ; 1.218]$} \\
\hline \multirow{2}{*}{ St. Dev. Pure Grams Per $\$ 100$} & 0.271 & 0.184 & 0.176 \\
\hline & {$[0.256 ; 0.279]$} & {$[0.156 ; 0.287]$} & {$[0.142 ; 0.180]$} \\
\hline \multirow{2}{*}{ Active Buyers, in Millions } & 3.431 & 1.000 & 1.073 \\
\hline & {$[3.312 ; 3.530]$} & {$[1.000 ; 1.000]$} & {$[1.042 ; 1.097]$} \\
\hline \multirow{2}{*}{ ACtive Sellers, in Millions } & 0.290 & 1.000 & 0.822 \\
\hline & {$[0.271 ; 0.295]$} & {$[1.000 ; 1.000]$} & {$[0.809 ; 0.844]$} \\
\hline \multirow{2}{*}{ FraCtion of MATChED Buyers (\%) } & 54.040 & 1.054 & 1.031 \\
\hline & {$[52.420 ; 55.100]$} & {$[1.024 ; 1.109]$} & {$[0.985 ; 1.053]$} \\
\hline \multirow{2}{*}{ Average Number of Purchases per Month } & 12.726 & 1.053 & 1.013 \\
\hline & {$[12.228 ; 13.389]$} & {$[1.038 ; 1.086]$} & {$[0.975 ; 1.028]$} \\
\hline \multirow{2}{*}{ Average Pure Grams Consumed per Month } & 9.464 & 1.082 & 1.042 \\
\hline & {$[9.057 ; 9.990]$} & {$[1.063 ; 1.115]$} & {$[1.000 ; 1.055]$} \\
\hline
\end{tabular}

Notes-This table reports market outcomes in the counterfactual cases in which buyers can observe drugs' purity before purchasing, expressed as ratios over the corresponding values in the baseline case. 95-percent confidence intervals in brackets.

participation. Second, the intensified competition among sellers reduces their profits relative to the baseline case, thereby decreasing sellers' participation. As a result, the buyer-seller ratio increases relative to the baseline case, attenuating some of the partial-equilibrium effects. Overall, the increase in the number of buyers (by approximately seven percent), along with the increase in their drug consumption (by approximately three percent), leads to an increase in the aggregate consumption of pure drugs by approximately 12 percent relative to the baseline case. However, some of the estimates are imprecise and, thus, we cannot rule out that the general-equilibrium effects fully offset the partial-equilibrium effects on individual purchases and consumption of active buyers.

The results reported in Table 4 can provide some insights into how outcomes differ between illegal and legal markets. Buyers' imperfect information is one key way in which our model captures an illegal market because, in legal markets for similar commodities (i.e., tea, coffee, cigarettes, alcohol), buyers are better (albeit perhaps not fully) informed about the quality of products that they are purchasing. While a full legalization counterfactual requires many additional assumptions - for example, on the destruction rate $\delta$, the efficiency of the matching process and the entry costs $K_{B}$ and $K_{S}$ - our analysis illustrates that the dispersion of quality of 
drugs will decrease and the average quality of drugs will increase if buyers gain better information. The reduction in quality dispersion may bring health benefits: Caulkins (2007) argues that greater variability in purity predicts overdoses because users inadvertently consume more pure drug than they intend to when they purchase drugs that are more pure than is typical; at the same time, the increase in average quality may increase aggregate consumption and, thus, may bring health costs. However, the results in Table 4 suggest that this aggregate increase will not be large, curbing these additional costs.

In summary, while we are not aware of any countries currently considering the legalization of crack cocaine, we believe that the economic mechanisms that this counterfactual highlights do not apply exclusively to the crack cocaine market, and, thus, this analysis could shed some light on how market outcomes would differ in the cases of substances that are currently being legalized.

\subsubsection{The Role of Penalties}

The United States has witnessed a large increase in policies and penalties on buyers and sellers of drugs in the last 30 years. The principal aim of these changes has been to disrupt the market for narcotics. Legal penalties on drug trade obviously affect sellers' flow costs $K_{S}$ and buyers' costs $K_{B}$. Thus, in this Section, we use our model to understand how these costs $K_{S}$ and $K_{B}$ affect sellers' and buyers' participation and, through it, market outcomes. ${ }^{27}$

We estimated our model on data from 2001-2003, when penalties were high; thus, we now perform two counterfactuals with lower costs. More specifically, in the first counterfactual, we decrease sellers' flow cost $K_{S}$ by 15 percent (i.e., from $\$ 1,500$ to $\$ 1,275$ ) relative to the baseline case of Section 4 with ex-ante unobservable quality; in the second one, we decrease buyers' flow cost $K_{B}$ by 15 percent relative to the baseline case. Online Appendix D reports the results with different changes to these flow costs.

Table 5 reports the quantitative values of market outcomes for the two counterfactuals cases, displaying interesting results. With lower penalties on sellers (i.e., a drop in $K_{S}$ ), the effect on market participation is intuitive: the number of sellers is approximately 30-percent higher. Thus, the rate $\alpha_{B}(\theta)$ at which buyers meet with new sellers is also higher.

The effect on the average offered quality, however, is quite striking: the average quality of a new purchase is lower, despite lower sellers' costs. Two factors account for this decrease: 1) A larger value of $\alpha_{B}(\theta)$ means that the match between a buyer and a seller is, on average, shorter-lived. In turn, this reduction lowers sellers' value of forming long-term relationships with

\footnotetext{
${ }^{27}$ Different policing interventions affect the market through different parameters. For example, prison sentencing guidelines more likely affect buyers' and sellers' costs, whereas police patrolling more likely affects the destruction rate $\delta$ and the rate $\gamma$ of repeat meetings. Table D1 in Online Appendix D reports on additional counterfactuals that consider a 20-percent decrease in $\delta$ and a 20-percent increase in $\gamma$, respectively.
} 
TABle 5: The Effect of Penalties

\begin{tabular}{|c|c|c|c|}
\hline & BASELINE & LOWER $K_{S}$ & LOWER $K_{B}$ \\
\hline \multirow{2}{*}{ FRACTION OF RIP-OFFS (\%) } & 15.862 & 1.678 & 1.162 \\
\hline & {$[13.646 ; 16.812]$} & {$[1.596 ; 1.811]$} & {$[1.080 ; 1.209]$} \\
\hline \multirow{2}{*}{ Average Pure Grams per $\$ 100$} & 0.616 & 0.866 & 0.972 \\
\hline & {$[0.597 ; 0.636]$} & {$[0.852 ; 0.877]$} & {$[0.950 ; 0.983]$} \\
\hline \multirow{2}{*}{ St. Dev. Pure Grams per $\$ 100$} & 0.271 & 1.195 & 1.062 \\
\hline & {$[0.256 ; 0.279]$} & {$[1.172 ; 1.235]$} & {$[1.029 ; 1.074]$} \\
\hline \multirow{2}{*}{ Active Buyers, in Millions } & 3.431 & 0.942 & 1.036 \\
\hline & {$[3.312 ; 3.530]$} & {$[0.929 ; 0.957]$} & {$[1.008 ; 1.042]$} \\
\hline \multirow{2}{*}{ Active Sellers, in Millions } & 0.290 & 1.304 & 1.036 \\
\hline & {$[0.271 ; 0.295]$} & {$[1.286 ; 1.324]$} & {$[1.008 ; 1.042]$} \\
\hline \multirow{2}{*}{ Fraction of Matched Buyers (\%) } & 54.040 & 0.973 & 0.996 \\
\hline & {$[52.420 ; 55.100]$} & {$[0.950 ; 1.032]$} & {$[0.955 ; 1.008]$} \\
\hline \multirow{2}{*}{ Average Number of Purchases Per Month } & 12.726 & 1.028 & 0.991 \\
\hline & {$[12.228 ; 13.389]$} & {$[1.000 ; 1.066]$} & {$[0.971 ; 1.015]$} \\
\hline \multirow{2}{*}{ Average Pure Grams Consumed per Month } & 9.464 & 1.004 & 0.988 \\
\hline & {$[9.057 ; 9.990]$} & {$[0.977 ; 1.044]$} & {$[0.969 ; 1.016]$} \\
\hline
\end{tabular}

Notes - This table reports market outcomes in the counterfactual cases in which buyers' cost $K_{B}$ and sellers' cost $K_{S}$ are 15-percent lower than in the baseline case, respectively, expressed as ratios over the corresponding values in the baseline case. 95-percent confidence intervals in brackets.

new buyers relative to the value of ripping them off; thus, the proportion of rip-offs increases. 2) The new sellers have higher average costs than sellers in the baseline case and, thus, sell low-quality drugs. Our quantitative exercise shows that, at the estimated parameter values, the proportion of sellers who specialize in rip-offs is approximately 70-percent higher (i.e., it increases to approximately 27 percentage points); the average offered quality is 14-percent lower; and the standard deviation is 20-percent higher, as compared to the baseline case. Moreover, the fact that relationships are shorter-lived is quantitatively more important than the fact that new sellers have higher costs in accounting for these changes in drug quality.

This decrease in drug quality decreases the number of active buyers in the market, whereas a higher meeting rate increases it. As a result of these opposing forces, the equilibrium number of buyers decreases, by less than six percent. Similarly, the lower quality of drugs implies that the fraction of matched buyers, their average number of purchases and their average pure quantity of crack consumed decrease. However, the magnitudes of all these changes are negligible and the confidence intervals imply most of them are not statistically different from the baseline results.

Similarly, a 15-percent reduction in buyers' participation cost $K_{B}$ leads to an increase in the number of buyers. Sellers' free-entry condition (A2) requires that the buyer-seller ratio equals that of the baseline case. Therefore, a larger number of sellers participate in the market than 
in the baseline case. Since these new sellers have higher costs, they have stronger incentives to make quick profits by selling $q=0$, decreasing the offered qualities. The last column of Table 5 reports the effects on the drug quality distribution: the fraction of rip-offs increases by 16.2 percent, the average drug quality decreases by 2.8 percent, and the standard deviation of drug quality increases by 6.2 percent, relative to the baseline case. However, the overall effects on the number of matched buyers, as well as on buyers' purchases and consumptions, are again negligible and statistically indistinguishable from the baseline results.

Overall, the results further highlight the role of buyers' incomplete information at the time of purchase, as sellers' moral hazard is fundamental to this, perhaps, counterintuitive relation between lower penalties - sellers' penalties, in particular - and lower quality. Specifically, when buyers have perfect information about quality in a frictional product market (e.g., the productmarket version of Burdett and Mortensen, 1998), an increase in buyers' meeting rate $\alpha_{B}(\theta)$ unambiguously increases competition among sellers, thereby leading to an improvement in buyers' terms of trade -i.e., an increase in the average offered quality. Therefore, if entry costs $K_{B}$ or $K_{S}$ were reduced in a market with full observability, quality and consumption would increase.

Moreover, the results reported in Table 5 indicate that changes in penalties have larger effects on the distribution of drugs offered on the market than on buyers' participation, as well as on their purchase and consumption patterns. Hence, these results highlight that increasing penalties may work to strengthen the long-term relationships between buyers and sellers that help overcome illegal markets' informational problems. Thus, our analysis suggests that increasing penalties may have contributed to the observed increased purity of retail drugs in the U.S. during the period 1990-2005; it is also consistent with the fact that the number of regular cocaine users does not seem to have changed considerably during the same period, according to data from the NSDUH and its predecessor, the National Household Survey on Drug Abuse (Choy et al., 1998; Substance Abuse and Mental Health Services Administration, 2006). Similarly, the UN office for Drugs and Crime reports that price-adjusted purity of drugs is lower in Europe than in the United States, ${ }^{28}$ whereas penalties for market participants - buyers, in particular - are lower in Europe than in the United States. Of course, there are potentially many other differences among U.S. markets over time, and between the U.S. and the European markets. Nonetheless, we find it interesting that our model conforms to these aggregate outcomes.

\section{Conclusions}

This paper develops a framework to understand illicit drug markets. We focus on two key characteristics of illegal markets: 1) the inability to verify/contract the quality of the good; and

\footnotetext{
${ }^{28}$ See https : //data. unodc .org.
} 
2) penalties on market participants. Buyers' inability to verify the quality of the good creates a trade-off for sellers. On the one hand, they can offer low-quality drugs, thus maximizing shortterm profits. On the other hand, they can offer high-quality drugs, thus inducing buyers to purchase from them again. In equilibrium, a distribution of quality levels persists.

We estimate the model using data on the U.S. market for crack cocaine. The model fits the data well. Our counterfactual analysis implies that buyers' inability to verify the quality of the good and, thus, sellers' moral hazard reduce the average and increase the dispersion of drug purity, thereby reducing drug consumption. Moreover, the estimated model implies that increasing penalties may increase the purity and the affordability of the drugs traded because it increases sellers' relative profitability of targeting loyal buyers versus first-time buyers.

We should point out that these results obtain in a model with important limitations and, thus, we believe that it can be enhanced in several ways. As we recount in Section 3, our crosssectional data impose some limitations on what our model can identify in the data, and richer panel-data on buyers and sellers would allow us to enrich our current framework. Specifically, multidimensional heterogeneity is difficult to identify with our data; thus, our model focuses on a single dimension of heterogeneity across buyers - i.e., their taste for drugs - and across sellers i.e., their cost of acquiring pure drugs - and restricts other parameters to be homogeneous across individuals - i.e., flow costs, the meeting rates, and the destruction rate - and across statesi.e., the new-meeting rate for matched and unmatched buyers. Similarly, we abstract from time-varying frequency of consumption, which would be relevant, for instance, if buyers become increasingly addicted over time and, thus, desire to consume more. Additionally, we abstract from referrals and word-of-mouth among buyers. Many structural search models share these features due to similar data constraints, and one objective of this paper is to adapt and to enrich these models to understand the key characteristics - i.e., imperfect observability and penaltiesof product markets for illicit substances. Furthermore, we let sellers commit to a single level of quality: a model that allows sellers to discriminate across their buyers or to vary quality over time would be difficult to identify with the available data, since we do not observe how sellers discriminate between first-time buyers and repeat buyers or whether they vary their strategy over time. ${ }^{29}$ Nonetheless, our theoretical framework delivers rich heterogeneity across sellers, and our empirical model successfully matches the large heterogeneity observed in the data.

For these main reasons, we view this paper as a first step in quantifying the role of imperfect observability in search markets. The empirical application clarifies the data requirements to estimate such a model and how the parameters are identified, and the estimation delivers a sense of the magnitudes involved, allowing us to assess which forces dominate. Nonetheless, we hope that the future availability of richer data will allow us to incorporate additional features of markets for illicit substances or, more generally, markets in which quality uncertainty is important.

\footnotetext{
${ }^{29}$ Sellers may face stronger incentives to offer time-varying quality levels in the case of addiction.
} 


\section{References}

Adda, J., B. McConnell, And I. Rasul (2014): "Crime and the Depenalization of Cannabis Possession: Evidence from a Policing Experiment," Journal of Political Economy, 122, 11301202.

Allen, J., R. Clark, and J.-F. Houde (2014): "Search frictions and market power in negotiated price markets," Working Paper 19883, National Bureau of Economic Research.

Arkes, J., R. L. Pacula, S. M. Paddock, J. P. Caulkins, and P. Reuter (2008): "Why the DEA Stride data are still useful for understanding drug markets," Working Paper 14224, National Bureau of Economic Research.

Becker, G. And K. Murphy (1988): "A Theory of Rational Addiction," Journal of Political Economy, 96, 675-700.

Becker, G. S., K. M. Murphy, And M. Grossman (2006): "The Market for Illegal Goods: the Case of Drugs," Journal of Political Economy, 114, 38-60.

Board, S. And M. Meyer-ter Vehn (2013): "Reputation for Quality," Econometrica, 81, 2381-2462.

Bontemps, C., J.-M. Robin, And G. Van den Berg (1999): "An empirical equilibrium job search model with search on the job and heterogeneous workers and firms," International Economic Review, 40, 1039-1074.

Bоoт, A. W. (2000): "Relationship Banking: What Do We Know?" Journal of Financial Intermediation, 9, 7-25.

Burdett, K. And D. Mortensen (1998): "Wage differentials, employer size, and unemployment," International Economic Review, 39, 257-273.

Caulkins, J. P. (2007): "Price and purity analysis for illicit drug: data and conceptual issues," Drug and Alcohol Dependence, 90, S61-S68.

Cebul, R. D., J. B. Rebitzer, L. J. Taylor, and M. E. Votruba (2011): "Unhealthy Insurance Markets: Search Frictions and the Cost and Quality of Health Insurance," The American Economic Review, 101, 1842-1871.

Choy, W., D. R. Gerstein, F. Gray, and R. Ghadialy (1998): National Household Survey on Drug Abuse: Main Findings, (1993), DIANE Publishing. 
Dave, D. (2008): "Illicit drug use among arrestees, prices and policy," Journal of Urban Economics, 63, 694-714.

Dranove, D. And G. Jin (2010): "Quality Disclosure and Certification: Theory and Practice," Journal of Economic Literature, 48, 935-963.

Eckstein, Z. And G. J. VAn Den Berg (2007): "Empirical labor search: A survey," Journal of Econometrics, 136, 531-564.

Fryer, R. G., P. S. Heaton, S. D. Levitt, and K. M. Murphy (2013): “Measuring Crack Cocaine and its Impact," Economic Inquiry, 51, 1651-1681.

Galenianos, M., R. L. Pacula, And N. Persico (2012): "A Search-Theoretic Model of the Retail Market for Illicit Drugs," The Review of Economic Studies, 79, 1239-1269.

GavazzA, A. (forthcoming): "An Empirical Equilibrium Model of a Decentralized Asset Market," Econometrica.

Hong, H. AND M. Shum (2006): "Using price distributions to estimate search costs," The RAND Journal of Economics, 37, 257-275.

Horowitz, J. L. (2001): "Should the DEA's STRIDE data be used for economic analyses of markets for illegal drugs?" Journal of the American Statistical Association, 96, 1254-1271.

Hortaçsu, A. And C. Syverson (2004): "Search costs, product differentiation, and welfare effects of entry: a case study of SP 500 index funds," The Quarterly Journal of Economics, $119,403-456$.

IIZUKA, T. (2012): "Physician agency and adoption of generic pharmaceuticals," The American Economic Review, 102, 2826-2858.

Imbens, G. W. And T. Lancaster (1994): "Combining Micro and Macro Data in Microeconometric Models," The Review of Economic Studies, 61, 655-680.

JACOBI, L. And M. Sovinsky (2016): "Marijuana on Main Street? Estimating Demand in Markets with Limited Access," The American Economic Review, 106, 2009-2045.

Kilmer, B., S. S. Everingham, J. P. Caulkins, G. Midgette, R. L. Pacula, P. H. Reuter, R. M. Burns, B. Han, and R. Lundberg (2014): "What America's Users Spend on Illegal Drugs," Research Reports RR-534-ONDCP, RAND Corporation.

Kuziemko, I. And S. Levitt (2004): "An empirical analysis of imprisoning drug offenders," Journal of Public Economics, 88, 2043-2066. 
Levitt, S. AND S. Venkatesh (2000): “An economic analysis of a drug-selling gang's finances," The Quarterly Journal of Economics, 115, 755-789.

Reuter, P. And J. P. Caulkins (2004): "Illegal 'lemons': price dispersion in cocaine and heroin markets," Bulletin on Narcotics, 56, 141-165.

Reuter, P., R. MacCoun, And P. Murphy (1990): "Money from Crime: A Study of the Economics of Drug Dealing in Washington, D.C." Research Reports R-3894-RF, RAND Corporation.

Substance Abuse and Mental Health Services Administration (2006): "Results from the 2005 National Survey on Drug Use and Health: National Findings," Tech. Rep. NSDUH Series H-30.

WildenbeEst, M. R. (2011): "An empirical model of search with vertically differentiated products," The RAND Journal of Economics, 42, 729-757.

\section{APPENDICES}

\section{A Proofs}

Proof of Proposition 2. We determine the reservation quality of an unmatched buyer by equating the value of remaining unmatched with the value of becoming matched at $R_{z}$ :

$$
\bar{V}_{z}=V_{z}\left(R_{z}\right)
$$

which implies equation (4). Integrating by parts, the value of being unmatched satisfies:

$$
r \bar{V}_{z}=\alpha_{B}(\theta)\left(z \int_{0}^{\bar{q}} x d F(x)+\int_{R_{z}}^{\bar{q}} V_{z}^{\prime}(x)(1-F(x)) d x-p\right)
$$

Differentiating equation (2) with respect to $q$, we obtain

$$
V_{z}^{\prime}(q)=\frac{\gamma z}{r+\delta+\alpha_{B}(\theta)(1-F(q))}
$$

Combining the previous two equations yields equation (3).

The value of participating in the market is negative for buyers who receive no utility from consuming and is strictly increasing in a buyer's marginal utility of consumption:

$$
\begin{aligned}
r \bar{V}_{0} & =-\alpha_{B}(\theta) p<0 \\
\frac{\partial r \bar{V}_{z}}{\partial z} & =\alpha_{B}(\theta)\left(\int_{0}^{\bar{q}} x d F(x)+\int_{R_{z}}^{\bar{q}} \frac{\gamma(1-F(x))}{r+\delta+\alpha_{B}(\theta)(1-F(x))} d x+\frac{p}{z^{2}} \frac{\alpha_{B}(\theta) \gamma\left(1-F\left(R_{z}\right)\right)}{r+\delta+\alpha_{B}(\theta)\left(1-F\left(R_{z}\right)\right)}\right)>0 .
\end{aligned}
$$


Since the value of participating is increasing in $z$, a buyer participates in the market only if his type is high enough.

Proof of Proposition 3. Fix a type- $z$ buyer and consider his value of participating in the market as a function of the buyer-seller ratio, $\theta$. If $\theta$ is large, type- $z$ buyer never meets with a seller $\left(\lim _{\theta \rightarrow \infty} \alpha_{B}(\theta)=\right.$ 0 ) and, thus, his value of participating is strictly below the entry cost:

$$
\lim _{\theta \rightarrow \infty} r \bar{V}_{z}=0<K_{B}
$$

The value of participating in the market is strictly decreasing in $\theta$ :

$$
\frac{\partial r \bar{V}_{z}}{\partial \theta}=\alpha_{B}^{\prime}(\theta)\left(z \int_{0}^{\bar{q}} x d F(x)-p\right)+\frac{z \alpha_{B}^{\prime}(\theta)(r+\delta)}{\alpha_{B}(\theta)^{2}} \int_{p / z}^{\bar{q}} \frac{\gamma(1-F(x))}{\left(\frac{r+\delta}{\alpha_{B}(\theta)}+1-F(x)\right)^{2}} d x<0 .
$$

If $\theta$ is small, a buyer meets with a seller arbitrarily often $\left(\lim _{\theta \rightarrow 0} \alpha_{B}(\theta)=\infty\right)$ and, thus, the value of participating satisfies:

$$
\lim _{\theta \rightarrow 0} r \bar{V}_{z}=\lim _{\theta \rightarrow 0} \alpha_{B}(\theta)\left(z \int_{0}^{\bar{q}} x d F(x)-p\right),
$$

and the buyer participates if:

$$
\lim _{\theta \rightarrow 0} \alpha_{B}(\theta)\left(z \int_{0}^{\bar{q}} x d F(x)-p\right)>K_{B}
$$

Hence, regardless of $\theta$, a type- $z$ buyer never participates if $z \int_{0}^{\bar{q}} x d F(x) \leq p$. As a result, there is no buyer entry $(B=0)$ when $\bar{z} \int_{0}^{\bar{q}} x d F(x) \leq p$, which proves part 1 of the Proposition.

When $\bar{z} \int_{0}^{\bar{q}} x d F(x)>p$, a type- $z$ buyer participates if $z \int_{0}^{\bar{q}} x d F(x)>p$ and if $\theta$ is low enough. Conversely, given $\theta$, there is a $z(\theta)$, with $z(\theta) \int_{0}^{\bar{q}} x d F(x)>p$, such that

$$
r \bar{V}_{z(\theta)}=K_{B}
$$

so that a buyer participates if and only if $z \geq z(\theta)$ and the measure of buyers equals $B=\bar{B}(1-\bar{M}(z(\theta)))$. Given $S$, this leads to

$$
\theta(z(\theta))=\frac{\bar{B}(1-\bar{M}(z(\theta)))}{S} .
$$

We now show that, given $S$ and $F(\cdot)$, there is a unique $z^{*}$ such that $z^{*}=z\left(\theta\left(z^{*}\right)\right)$. We show that as $z^{*}$ increases, the participation value of the marginal buyer type increases after taking into account the effect on $\theta$ :

$$
\frac{d r \bar{V}_{z^{*}}}{d z^{*}}=\frac{\partial r \bar{V}_{z^{*}}}{\partial z^{*}}+\frac{\partial r \bar{V}_{z^{*}}}{\partial \theta}\left(-\bar{B} \bar{M}^{\prime}\left(z^{*}\right)\right)>0 .
$$

Therefore, there is a unique $z^{*}$ such that the unmatched value of the marginal buyer is exactly equal to $K_{B}$ and is defined by equation (5).

This completes the proof of parts 2 and 3.

Proof of Lemma 5. For $q \in[0, \underline{R})$, we have $t(q)=t_{N}$, which implies that $\pi_{c}(0)>\pi_{c}(q)$ for $q \in(0, \underline{R})$. Therefore, either $q=0$, or $q \geq \underline{q}$ for some $\underline{q} \geq \underline{R}$ and $F$ is constant (and, hence, continuous) on [0, $q$ ]. If $\underline{q}>\bar{R}$, then $t(q)=t(\bar{R})$ for $\bar{q} \in[\bar{R}, q]$, which implies that $\pi_{c}(\bar{R})>\pi_{c}(q)$ for $q \in(\bar{R}, q]$. Therefore, $\underline{q} \leq \bar{R}$. Standard arguments (as in Burdett and Mortensen, 1998) prove that $F$ is continuous and its support is connected on $[\underline{q}, \bar{q}]$.

Proof of Proposition 6. We determine the number of unmatched buyers and their type distribution using the fact that, in steady state, the flow of buyers into and out of the matched state must be equal. 
Let $n(R)$ denote the number of buyers who are unmatched and whose type is less than $R$. The total number of unmatched buyers is $n(\bar{R}) \equiv \bar{n}$. An unmatched buyer of type $R$ becomes matched after transacting with a seller who offers above-reservation quality, which occurs at rate $\alpha_{B}(\theta)(1-F(R))$. A matched buyer exits the matched state when his match is exogenously destroyed, which occurs at rate $\delta$. In steady state:

$$
n^{\prime}(R) \alpha_{B}(\theta)(1-(F(R)))=\delta\left(B H^{\prime}(R)-n^{\prime}(R)\right) \Rightarrow n^{\prime}(R)=\frac{\delta B H^{\prime}(R)}{\delta+\alpha_{B}(\theta)(1-F(R))} .
$$

Hence, the mass $n(R)$ satisfies:

$$
n(R)=\int_{\underline{R}}^{R} \frac{B \delta}{\delta+\alpha_{B}(\theta)(1-F(x))} d H(x),
$$

and, thus, the mass of matched buyers is:

$$
B-\bar{n}=B\left(1-\int_{\underline{R}}^{\bar{R}} \frac{\delta}{\delta+\alpha_{B}(\theta)(1-F(x))} d H(x)\right)=\int_{\underline{R}}^{\bar{R}} \frac{B \alpha_{B}(\theta)(1-F(x))}{\delta+\alpha_{B}(\theta)(1-F(x))} d H(x) .
$$

Let $G(\cdot)$ denote the received quality distribution for matched buyers. The mass of matched buyers receiving quality up to $q$ equals $(B-\bar{n}) G(q)$. An unmatched type- $R$ buyer flows into this group if $R \leq q$ and he samples a seller who offers quality less than $q$, which occurs at rate $\alpha_{B}(\theta)(F(q)-F(R))$. A buyer flows out of this group if the match is exogenously destroyed or if he samples a new seller whose quality if greater than $q$, which occurs at rate $\delta+\alpha_{B}(\theta)(1-F(q))$. Equating these flows yields:

$$
\begin{aligned}
\alpha_{B}(\theta) \int_{\underline{R}}^{q}(F(q)-F(x)) d n(x) & =(B-\bar{n}) G(q)\left(\delta+\alpha_{B}(\theta)(1-F(q))\right) \\
\Rightarrow(B-\bar{n}) G(q) & =\frac{\alpha_{B}(\theta) B \delta \int_{\underline{R}}^{q} \frac{F(q)-F(x)}{\delta+\alpha_{B}(\theta)(1-F(x))} d H(x)}{\delta+\alpha_{B}(\theta)(1-F(q))} .
\end{aligned}
$$

Thus, $G^{\prime}(q)$ satisfies (we assume, and later verify, that $F$ is differentiable):

$$
(B-\bar{n}) G^{\prime}(q)=\frac{\alpha_{B}(\theta) B \delta F^{\prime}(q) H(q)}{\left(\delta+\alpha_{B}(\theta)(1-F(q))\right)^{2}} .
$$

The number of buyers who are matched with a seller offering $q$ is $(B-\bar{n}) G^{\prime}(q)$, and the number of sellers offering quality $q$ is $S F^{\prime}(q)$. Hence, the buyer-seller ratio at quality $q$ is:

$$
l(q)=\frac{(B-\bar{n}) G^{\prime}(q)}{S F^{\prime}(q)},
$$

which implies that the flow of transactions from regular buyers is:

$$
t_{R}(q)=\frac{\gamma \alpha_{B}(\theta) \theta \delta H(q)}{\left(\delta+\alpha_{B}(\theta)(1-F(q))\right)^{2}} .
$$

Combining results completes the proof of Proposition 6.

Proof of Proposition 7. First, we show that $F(0)=1$ cannot occur in equilibrium. By contradiction, suppose that all participating sellers offer $q=0$. In equilibrium, their profits are equal to the entry cost $K_{S}$ and they are given by $\pi_{c}(0)=\alpha_{B}(\theta) \theta p$. Consider a seller with cost $c^{\prime}$ who deviates and offers 
$q^{\prime}=\bar{R}$. His profits are

$$
\pi_{c^{\prime}}(\bar{R})=\alpha_{B}(\theta) \theta\left(1+\frac{\gamma \delta}{\delta^{2}}\right)\left(p-c^{\prime} \bar{R}\right)
$$

which is strictly greater than $K_{S}$ for $c^{\prime}$ close enough to zero. Since $c \in[0, \bar{c}]$, such a type exists and strictly prefers to participate in the market, providing the desired profit-increasing deviation.

Second, quality and profits are decreasing in seller-type. Fix sellers 1 and 2 with $c_{1}>c_{2}$ who offer $q_{1}$ and $q_{2}$. Suppose that $\underline{R} \leq q_{2} \leq q_{1}$. By revealed preference:

$$
\begin{aligned}
& \left(p-c_{1} q_{1}\right) t\left(q_{1}\right) \geq\left(p-c_{1} q_{2}\right) t\left(q_{2}\right) \Rightarrow p\left(t\left(q_{1}\right)-t\left(q_{2}\right)\right) \geq c_{1}\left(t\left(q_{1}\right) q_{1}-t\left(q_{2}\right) q_{2}\right), \\
& \left(p-c_{2} q_{2}\right) t\left(q_{2}\right) \geq\left(p-c_{2} q_{1}\right) t\left(q_{1}\right) \Rightarrow p\left(t\left(q_{1}\right)-t\left(q_{2}\right)\right) \leq c_{2}\left(t\left(q_{1}\right) q_{1}-t\left(q_{2}\right) q_{2}\right),
\end{aligned}
$$

which, combined with the assumption $c_{1}>c_{2}$, yields a contradiction. We have shown that $q_{2} \geq \underline{R} \Rightarrow$ $q_{2}>q_{1}$. Seller 2's profits are higher because $\pi_{c_{2}}\left(q_{2}\right) \geq \pi_{c_{2}}\left(q_{1}\right)>\pi_{c_{1}}\left(q_{1}\right)$. Going through similar steps proves that $q_{2}=0 \Rightarrow q_{1}=0$. Finally, if $q=0$ is offered, then there is a marginal seller $c^{*}>0$ such that sellers with $c>c^{*}$ offer $q=0$, and sellers with $c \leq c^{*}$ offer $q>0$.

The free-entry condition implies that the profits of the marginal seller type are equal to $K_{S}$; all sellers with $c<c^{*}$ participate in the market; and the measure of sellers who offer positive quality is $S_{+}\left(c^{*}\right)=\bar{S} \bar{D}\left(c^{*}\right)$. Denote the measure of sellers who participate in the market and offer $q=0$ by $S_{0}$, where $S_{0} \geq 0$. Their profits do not depend on their type and satisfy

$$
p \alpha_{S}\left(\frac{B}{S_{+}\left(c^{*}\right)+S_{0}}\right) \leq K_{S},
$$

which holds with equality if $S_{0}>0$.

We characterize $c^{*}$ and $S_{0}$ in three steps. 1) We characterize the optimal quality offered by the marginal seller conditional on his type and $S_{0}$. 2) Given $S_{0}$, we show that there is a unique marginal type $c^{*}$ whose profits equal $K_{S}$. 3) We characterize $S_{0}$.

The profits of the seller who offers the lowest positive quality (the marginal seller) satisfy:

$$
\underline{\pi}_{c}(q)=\alpha_{S}(\theta)(p-c q)\left(1+\frac{\gamma \delta H(q)}{\left(\delta+\alpha_{B}(\theta)(1-F(0))\right)^{2}}\right)
$$

where $F(0)=\frac{S_{0}}{S}$ and $S=S_{+}(c)+S_{0}$.

Denote the optimal choice for a type- $c$ seller who offers the lowest positive quality by $\underline{q}(c)$. The optimal lowest positive quality is given by the root of:

$$
\underline{\pi}_{c}^{\prime}(q)=\alpha_{S}(\theta)\left[-c\left(1+\frac{\gamma \delta H(q)}{\left(\delta+\alpha_{B}(\theta)(1-F(0))\right)^{2}}\right)+(p-c q) \frac{\gamma \delta H^{\prime}(q)}{\left(\delta+\alpha_{B}(\theta)(1-F(0))\right)^{2}}\right],
$$

where the the log-concavity of $H(\cdot)$ guarantees that the solution is unique:

$$
\underline{\pi}_{c}^{\prime \prime}(q)=\alpha_{S}(\theta) \frac{-2 c \gamma \delta H^{\prime}(q)+(p-c q) \gamma \delta H^{\prime \prime}(q)}{\left(\delta+\alpha_{B}(\theta)(1-F(0))\right)^{2}}<0 .
$$

Notice that it is never optimal to offer $q \in(0, \underline{R})$ or $q>\bar{R}$.

We show that, given $S_{0}$, there is a unique marginal type $c^{*}$ such that his profits are exactly equal 
to $K_{S}$ when he offers $\underline{q}\left(c^{*}\right)$. Notice that:

$$
\begin{aligned}
\lim _{c \rightarrow 0} \underline{\pi}_{c}(\underline{q}(c)) & =\lim _{c \rightarrow 0} \alpha_{S}\left(\frac{B}{S_{0}}\right) p t(\underline{q}(c))>K_{S}, \\
\lim _{c \rightarrow \bar{c}} \underline{\pi}_{c}(\underline{q}(c)) & =\alpha_{S}\left(\frac{B}{\bar{S}}\right) \lim _{c \rightarrow \infty}(p-c \underline{q}(c)) t(\underline{q}(c))<K_{S}, \\
\frac{d \underline{\pi}_{c}(\underline{q}(c))}{d c} & =\underline{\pi}_{c}^{\prime}(\underline{q}(c)) \frac{d \underline{q}(c)}{d c}+\frac{\partial \underline{\pi}_{c}(\underline{q}(c))}{\partial c}+\frac{\partial \underline{\pi}_{c}}{\partial \theta} \frac{d \theta}{d c^{*}}<0,
\end{aligned}
$$

where we assume that $\bar{c}$ is large enough. In the last equation, the first term is zero by the envelope condition; the second term is negative because higher costs reduce margins; and the third term is negative because an increase in $c^{*}$ increases the measure of participating sellers, which reduces $\alpha_{S}$. This proves that there is a unique $c^{*}$, given $S_{0}$, such that $\underline{\pi}\left(\underline{q}\left(c^{*}\right)\right)=K_{S}$.

To determine $S_{0}$, we show that an increase in $\bar{S}$ leads to an increase in $S_{+}\left(c^{*}\right)=\bar{S} \bar{D}\left(c^{*}\right)$ and to a decrease in $\theta$. By contradiction, suppose the opposite. Hence, $c^{*}$ drops and, thus, the (new) marginal type's profits are strictly greater than $K_{S}$, as he has lower costs and faces fewer competing sellers, yielding a contradiction.

Define $\hat{S}$ as the measure of potential sellers such that equation (A2) holds with equality when $\bar{S}=\hat{S}$ and $S_{0}=0$. If $\bar{S} \geq \hat{S}$, then $S_{0}=0, S=S_{+}\left(c^{*}\right)$, and $c^{*}$ is determined by equating (A4) to zero and (A3) to $K_{S}$. If $\bar{S}<\hat{S}$, then the measure of sellers $S$ is determined by $\alpha_{S}\left(\frac{B}{S}\right) p=K_{S}$; $c^{*}$ is determined by equating (A4) to zero and (A3) to $K_{S}$; and $S_{0}=S-S_{+}\left(c^{*}\right)$.

We now determine $q^{*}(c)$ for $c<c^{*}$. We assume that an optimal schedule $q^{*}(c)$ exists, and we rewrite the profits of a type- $c$ seller as if he decides which other type $c^{\prime}$ to imitate rather than which quality to offer-i.e., to imitate type $c^{\prime}$, who offers quality $q^{\prime}=q^{*}\left(c^{\prime}\right)$. Hence:

$$
\pi_{c}\left(c^{\prime}\right)=\alpha_{B}(\theta) \theta\left(p-c q^{*}\left(c^{\prime}\right)\right)\left(1+\frac{\gamma \delta H\left(q^{*}\left(c^{\prime}\right)\right)}{\left(\delta+\alpha_{B}(\theta)(1-F(0)) D\left(c^{\prime}\right)\right)^{2}}\right),
$$

where $D(c)=\frac{\bar{D}(c)}{\bar{D}\left(c^{*}\right)}$ is the distribution of sellers who offer positive quality.

Differentiating profits with respect to $c^{\prime}$, we obtain:

$$
\begin{aligned}
\pi_{c}^{\prime}\left(c^{\prime}\right)= & \alpha_{B}(\theta) \theta c\left(-q^{*} \prime\left(c^{\prime}\right)\left(1+\frac{\gamma \delta H\left(q^{*}\left(c^{\prime}\right)\right)}{\left(\delta+\alpha_{B}(\theta)(1-F(0)) D\left(c^{\prime}\right)\right)^{2}}\right)+\right. \\
& \left.\left(\frac{p}{c}-q^{*}\left(c^{\prime}\right)\right) \gamma \delta \frac{H^{\prime}\left(q^{*}\left(c^{\prime}\right)\right) q^{*}\left(c^{\prime}\right)\left(\delta+\alpha_{B}(\theta)(1-F(0)) D\left(c^{\prime}\right)\right)-H\left(q^{*}\left(c^{\prime}\right)\right) 2 \alpha_{B}(\theta)(1-F(0)) D^{\prime}\left(c^{\prime}\right)}{\left(\delta+\alpha_{B}(\theta)(1-F(0)) D\left(c^{\prime}\right)\right)^{3}}\right) .
\end{aligned}
$$

By construction, $c^{\prime}=c$ maximizes profits. Setting the derivative to zero, we obtain:

$$
q^{*} \prime(c)=-\frac{2 \gamma \delta\left(\frac{p}{c}-q^{*}(c)\right) H\left(q^{*}(c)\right) \alpha_{B}(\theta)(1-F(0)) D^{\prime}(c)}{\left(\delta+\alpha_{B}(\theta)(1-F(0)) D(c)\right)\left[\left(\delta+\alpha_{B}(\theta)(1-F(0)) D(c)\right)^{2}+\gamma \delta H\left(q^{*}(c)\right)-\gamma \delta\left(\frac{p}{c}-q^{*}(c)\right) H^{\prime}\left(q^{*}(c)\right)\right]} .
$$

This differential equation and the initial condition $q^{*}\left(c^{*}\right)=\underline{q}\left(c^{*}\right)$ determine $q^{*}(c)$ for $c<c^{*}$.

\section{B Selection into ADAM}

In this Appendix, we derive the density of $\log$ preferences $z$ in the ADAM dataset.

We denote the events of arrest by $A$, of no arrest by $A^{C}$, of using drugs (i.e., participation in the market) by $U$, and of not using drugs by $U^{C}$. The fractions of agents who use drugs and do not use 
drugs are, respectively:

$$
\begin{aligned}
P(U) & =(1-\lambda)\left(1-M\left(z^{*}\right)\right), \\
P\left(U^{C}\right) & =\lambda+(1-\lambda) M\left(z^{*}\right),
\end{aligned}
$$

where we recall that $M(\cdot)$ is the distribution of preferences $z$, which we assume to be lognormal with parameters $\mu_{z}$ and $\sigma_{z}$, and $z^{*}$ is the preference of the marginal buyer.

An individual who does not use drugs is arrested if $\eta \geq 0$, where $\eta$ is normally distributed with mean $\mu_{\eta}$ and variance $\sigma_{\eta}^{2}$. Therefore, the arrest rate of non-users is:

$$
P\left(A \mid U^{C}\right)=1-\Phi\left(\frac{-\mu_{\eta}}{\sigma_{\eta}}\right)=\Phi\left(\frac{\mu_{\eta}}{\sigma_{\eta}}\right),
$$

where $\Phi(\cdot)$ denotes the cumulative distribution function of the standard normal distribution.

A drug user with taste $z$ is arrested if $\log (z)+\eta \geq 0$. Therefore, the arrest rate of drug users is:

$$
\begin{aligned}
P(A \mid U) & =\operatorname{Pr}\left(\log (z)+\eta \geq 0 \mid z \geq z^{*}\right) \\
& =\frac{1}{1-\Phi\left(\frac{\log \left(z^{*}\right)-\mu_{z}}{\sigma_{z}}\right)} \int_{\log \left(z^{*}\right)}^{\infty} \frac{1}{\sigma_{z}} \phi\left(\frac{\log (z)-\mu_{z}}{\sigma_{z}}\right) \Phi\left(\frac{\log (z)+\mu_{\eta}}{\sigma_{\eta}}\right) d \log (z) .
\end{aligned}
$$

Thus, the aggregate arrest rate is:

$$
\begin{aligned}
P(A) & =\left(\lambda+(1-\lambda) \Phi\left(\frac{\log \left(z^{*}\right)-\mu_{z}}{\sigma_{z}}\right)\right) \Phi\left(\frac{\mu_{\eta}}{\sigma_{\eta}}\right) \\
& +(1-\lambda) \int_{\log \left(z^{*}\right)}^{\infty} \frac{1}{\sigma_{z}} \phi\left(\frac{\log (z)-\mu_{z}}{\sigma_{z}}\right) \Phi\left(\frac{\log (z)+\mu_{\eta}}{\sigma_{\eta}}\right) d \log (z) .
\end{aligned}
$$

The fraction of drug users among those arrested (i.e., in ADAM) equals:

$$
\begin{gathered}
P(U \mid A)=\frac{P(A \mid U) P(U)}{P(A)} \\
=\frac{\int_{\log \left(z^{*}\right)}^{\infty} \frac{1}{\sigma_{z}} \phi\left(\frac{\log (z)-\mu_{z}}{\sigma_{z}}\right) \Phi\left(\frac{\log (z)+\mu_{\eta}}{\sigma_{\eta}}\right) d \log (z)}{\left(\frac{\lambda}{1-\lambda}+\Phi\left(\frac{\log \left(z^{*}\right)-\mu_{z}}{\sigma_{z}}\right)\right) \Phi\left(\frac{\mu_{\eta}}{\sigma_{\eta}}\right)+\int_{\log \left(z^{*}\right)}^{\infty} \frac{1}{\sigma_{z}} \phi\left(\frac{\log (z)-\mu_{z}}{\sigma_{z}}\right) \Phi\left(\frac{\log (z)+\mu_{\eta}}{\sigma_{\eta}}\right) d \log (z)} .
\end{gathered}
$$

Similarly, the fraction of drug users among those not arrested (i.e., in the NSDUH) equals:

$$
\begin{gathered}
P\left(U \mid A^{C}\right)=\frac{P\left(A^{C} \mid U\right) P(U)}{P\left(A^{C}\right)} \\
=\frac{\int_{\log \left(z^{*}\right)}^{\infty} \frac{1}{\sigma_{z}} \phi\left(\frac{\log (z)-\mu_{z}}{\sigma_{z}}\right)\left(1-\Phi\left(\frac{\log (z)+\mu_{\eta}}{\sigma_{\eta}}\right)\right) d \log (z)}{\frac{1}{1-\lambda}-\left(\frac{\lambda}{1-\lambda}+\Phi\left(\frac{\log \left(z^{*}\right)-\mu_{z}}{\sigma_{z}}\right)\right) \Phi\left(\frac{\mu_{\eta}}{\sigma_{\eta}}\right)-\int_{\log \left(z^{*}\right)}^{\infty} \frac{1}{\sigma_{z}} \phi\left(\frac{\log (z)-\mu_{z}}{\sigma_{z}}\right) \Phi\left(\frac{\log (z)+\mu_{\eta}}{\sigma_{\eta}}\right) d \log (z)} .
\end{gathered}
$$

Finally, the density of log preferences of drug users in ADAM satisfies:

$$
\frac{\phi\left(\frac{\log (z)-\mu_{z}}{\sigma_{z}}\right) \Phi\left(\frac{\log (z)+\mu_{\eta}}{\sigma_{\eta}}\right)}{\int_{\log \left(z^{*}\right)}^{\infty} \phi\left(\frac{\log (z)-\mu_{z}}{\sigma_{z}}\right) \Phi\left(\frac{\log (z)+\mu_{\eta}}{\sigma_{\eta}}\right) d \log (z)} \text { for } z \geq z^{*} .
$$

In practice, the density (B3) is the density of buyers' preferences weighted by the probability $\Phi\left(\frac{\log (z)+\mu_{\eta}}{\sigma_{\eta}}\right)$ of being in ADAM. 


\section{ONLINE APPENDICES (Not For Publications)}

\section{Observable Drug Purity}

In this Appendix, we prove the existence of equilibrium and derive its characterization when buyers observe drug quality before making a purchase. The analysis mirrors that of the baseline model.

\section{C.1 The Buyers}

We derive buyers' actions $(H(\cdot)$ and $B)$ taking as given sellers' behavior $(F(\cdot)$ and $S)$.

A type- $z$ buyer takes as given other agents' actions and makes two decisions when meeting a new seller: whether to consume (reservation $\hat{R}_{z}$ ) and, if so, whether to match (reservation $R_{z}$ if unmatched and $q$ if matched with a $q$-seller). Notice that a buyer will never match with a seller whose product he does not want to consume. The value functions of a type- $z$ buyer satisfy:

$$
\begin{aligned}
r \bar{V}_{z} & =\alpha_{B}(\theta) \int_{0}^{\bar{q}} \max \left[z x-p+\max \left[V_{z}(x)-\bar{V}_{z}, 0\right], 0\right] d F(x), \\
r V_{z}(q) & =\gamma(z q-p)+\alpha_{B}(\theta) \int_{0}^{\bar{q}} \max \left[z x-p+\max \left[V_{z}(x)-V_{z}(q), 0\right], 0\right] d F(x)+\delta\left(\bar{V}_{z}-V_{z}(q)\right) .
\end{aligned}
$$

Comparing the static costs and benefits of consumption and equating the value functions deliver the reservation quality for becoming matched:

$$
R_{z}=\hat{R}_{z}=\frac{p}{z}
$$

Thus, we can rewrite the value functions as follows:

$$
\begin{aligned}
r \bar{V}_{z} & =\alpha_{B}(\theta) \int_{\frac{p}{z}}^{\bar{q}}\left(z x-p+V_{z}(x)-\bar{V}_{z}\right) d F(x), \\
r V_{z}(q) & =\gamma(z q-p)+\alpha_{B}(\theta)\left(\int_{\frac{p}{z}}^{\bar{q}}(z x-p) d F(x)+\int_{q}^{\bar{q}}\left(V_{z}(x)-V_{z}(q)\right) d F(x)\right)+\delta\left(\bar{V}_{z}-V_{z}(q)\right) .
\end{aligned}
$$

Using integration by parts, the value of being unmatched satisfies:

$$
\begin{aligned}
r \bar{V}_{z} & =\alpha_{B}(\theta)\left(\left.\left(z q-p+V_{z}(q)-\bar{V}_{z}\right) F(q)\right|_{R_{z}} ^{\bar{q}}-\int_{R_{z}}^{\bar{q}}\left(z+V_{z}^{\prime}(x)\right) F(x) d x\right) \\
& =\alpha_{B}(\theta)\left(z\left(\bar{q}-R_{z}\right)+V_{z}(\bar{q})-V_{z}\left(R_{z}\right)-\int_{\frac{p}{z}}^{\bar{q}}\left(z+V_{z}^{\prime}(x)\right) F(x) d x\right)
\end{aligned}
$$

where we used $R_{z}=\frac{p}{z}$ and $\bar{V}_{z}=V_{z}\left(R_{z}\right)$ in the last equality. Using the fundamental theorem of calculus, we obtain:

$$
\begin{aligned}
r \bar{V}_{z} & =\alpha_{B}(\theta)\left(\int_{\frac{p}{z}}^{\bar{q}}\left(z+V_{z}^{\prime}(x)\right) d x-\int_{\frac{p}{z}}^{\bar{q}}\left(z+V_{z}^{\prime}(x)\right) F(x) d x\right) \\
& =\alpha_{B}(\theta) \int_{\frac{p}{z}}^{\bar{q}}\left[z+V_{z}^{\prime}(q)\right](1-F(x)) d x .
\end{aligned}
$$


Differentiating the value of being matched with respect to $q$ and rearranging, we obtain:

$$
V_{z}^{\prime}(q)=\frac{\gamma z}{r+\delta+\alpha_{B}(\theta)(1-F(q))}
$$

Hence, combining the previous two equations, we obtain:

$$
r \bar{V}_{z}=z \alpha_{B}(\theta) \int_{\frac{p}{z}}^{\bar{q}}\left(1+\frac{\gamma}{r+\delta+\alpha_{B}(\theta)(1-F(x))}\right)(1-F(x)) d x .
$$

We now determine whether a buyer of type $z$ participates in the market, taking as given other agents' actions summarized by $\{F(\cdot), \theta\}$.

Notice that:

$$
\begin{aligned}
r \bar{V}_{0}= & -\alpha_{B}(\theta) p<0, \\
\frac{\partial r \bar{V}_{z}}{\partial z}= & \alpha_{B}(\theta) \int_{0}^{\bar{q}} x d F(x)+\alpha_{B}(\theta) \int_{R_{z}}^{\bar{q}} \frac{\gamma(1-F(x))}{r+\delta+\alpha_{B}(\theta)(1-F(x))} d x+ \\
& \frac{p}{z^{2}} \frac{\alpha_{B}(\theta) \gamma\left(1-F\left(R_{z}\right)\right)}{r+\delta+\alpha_{B}(\theta)\left(1-F\left(R_{z}\right)\right)}>0,
\end{aligned}
$$

which prove that there exists a $\tilde{z}(F, \theta)$ such that a buyer participates if and only if $z \geq \tilde{z}(F, \theta)$.

Aggregating the entry decision across buyers, we obtain:

Proposition 10 Given $F(\cdot)$ and $S$ :

1. If $\frac{p}{\bar{q}} \geq \bar{z}$, then there is no buyer entry: $B=0$.

2. If $\frac{p}{\bar{q}}<\bar{z}$, then there is a unique buyer type $z^{* *} \leq \bar{z}$ such that all buyers with $z>z^{* *}$ participate in the market and all buyers with $z \leq z^{* *}$ do not.

3. The marginal buyer type is given by the solution to:

$$
z^{* *} \alpha_{B}(\theta) \int_{\frac{p}{z^{* *}}}^{\bar{q}}\left(1+\frac{\gamma}{r+\delta+\alpha_{B}(\theta)(1-F(q))}\right)(1-F(q)) d q=K_{B}
$$

Proof. Buyers with $z \leq \frac{p}{\bar{q}}$ get no benefit from participating in the market and never enter. Consider a buyer with $z>\frac{p}{\bar{q}}$. Whether this buyer enters the market depends on how quickly he trades, which, in turn, depends on the buyer-seller ratio $\theta$. We have:

$$
\begin{aligned}
\lim _{\theta \rightarrow \infty} r \bar{V}_{z} & =0<K_{B}, \\
\lim _{\theta \rightarrow 0} r \bar{V}_{z} & =\lim _{\theta \rightarrow 0} \alpha_{B}(\theta) \int_{\frac{p}{z}}^{\bar{q}}(1-F(x)) d x>K_{B}, \\
\frac{\partial r \bar{V}_{z}}{\partial \theta} & =z \int_{\frac{p}{z}}^{\bar{q}}\left(\alpha_{B}^{\prime}(\theta)+\frac{\alpha_{B}^{\prime}(\theta)(r+\delta)}{\alpha_{B}(\theta)^{2}} \frac{\gamma}{\left(\frac{r+\delta}{\alpha_{B}(\theta)}+1-F(x)\right)^{2}}\right)(1-F(x)) d x<0 .
\end{aligned}
$$

Therefore, for each buyer of type $z$ with $z>\frac{p}{\bar{q}}$, there is a unique $\theta(z)$ such that he participates if $\theta \leq \theta(z)$ and stays out otherwise. Hence, the measure of buyers in the market is:

$$
B=\bar{B}(1-\bar{M}(z(\theta))) .
$$


Given $S$, this leads to

$$
\theta(z(\theta))=\frac{\bar{B}(1-\bar{M}(z(\theta)))}{S} .
$$

We now show that, given $S$ and $F(\cdot)$, there is a unique $z^{* *}$ such that $z^{* *}=z\left(\theta\left(z^{* *}\right)\right)$. We show that as $z^{* *}$ increases, the participation value of the marginal type increases after taking into account the effect on $\theta$ :

$$
\frac{d r \bar{V}_{z^{* *}}}{d z^{* *}}=\frac{\partial r \bar{V}_{z^{* *}}}{\partial z^{* *}}+\frac{\partial r \bar{V}_{z^{* *}}}{\partial \theta}\left(-\bar{B} \bar{M}^{\prime}\left(z^{* *}\right)\right)>0
$$

Therefore, there is a unique $z^{* *}$ such that the unmatched value of the marginal buyer equals $K_{B}$, as in equation $(\mathrm{C} 1)$.

Corollary 11 The number of buyers who participate in the market is $B=\bar{B}\left(1-\bar{M}\left(z^{* *}\right)\right)$.

As in the baseline model, the distribution of reservation qualities obtains:

$$
H(R)= \begin{cases}0 & \text { if } R \leq \underline{R}, \\ \frac{1-\bar{M}\left(\frac{p}{R}\right)}{1-\bar{M}\left(z^{* *}\right)} & \text { if } R \in[\underline{R}, \bar{R}], \\ 1 & \text { if } R \geq \bar{R}\end{cases}
$$

where $\underline{R}=R_{z}=\frac{p}{z}$ and $\bar{R}=R_{z^{* *}}=\frac{p}{z^{* *}}$.

\section{C.2 The Sellers}

We derive sellers' actions, taking as given the measure of buyers who participate $B$ and the distribution of reservation qualities $H(\cdot)$.

Free entry determines the measure $S$ and type distribution $D(\cdot)$ of sellers in the market, subject to flow participation cost $K_{S}$. The problem of a seller of type $c$ is to choose a level of quality $q^{* *}(c)$ that maximizes her steady-state profits, which depend on the margin per transaction $(p-c q)$ and the steady-state flow of transactions $(t(q))$ :

$$
\pi_{c}(q)=(p-c q) t(q)
$$

We first derive some necessary conditions on the distribution of offered qualities:

Lemma 12 In equilibrium, the quality distribution $F$ has support on a subset of $[\underline{q}, \bar{q}]$, where $\underline{q} \in[\underline{R}, \bar{R}]$, and is continuous on $[0, \bar{q}]$.

Proof. For $q \in[0, \underline{R})$, we have $t(q)=0$ and, therefore, $q \geq \underline{q}$ for some $\underline{q} \geq \underline{R}$. If $\underline{q}>\bar{R}$, then $t(q)=t(\bar{R})$ for $q \in[\bar{R}, \underline{q}]$, which implies that $\pi_{c}(\bar{R})>\pi_{c}(q)$ for $q \in(\bar{R}, q]$. Therefore, $\underline{q} \leq \bar{R}$. The previous point proves that $F$ is constant (and, hence, continuous) on $[0, \underline{q}]$. Standard arguments (as in Burdett and Mortensen, 1998) prove continuity on $[\underline{q}, \bar{q}]$.

We take $H(\cdot), F(\cdot)$ and $\theta$ as given and calculate the steady-state profits that a type- $c$ seller would enjoy for any quality $q$. The main result is summarized in the next proposition.

Proposition 13 The steady-state profits of a seller of type $c$ who offers quality q are:

$$
\pi_{c}(q)=\alpha_{B}(\theta) \theta H(q)(p-c q)\left(1+\frac{\gamma \delta}{\left(\delta+\alpha_{B}(\theta)(1-F(q))\right)^{2}}\right), \quad q \geq \underline{q} .
$$


Proof. To determine profits, we need to first determine the flow of a seller's transactions as a function of the quality that she offers. The rate at which an individual seller transacts with a new buyer equals the meeting rate times the probability that the seller's quality is above the buyer's reservation:

$$
t_{N}(q)=\alpha_{S}(\theta) H(q)=\theta \alpha_{B}(\theta) H(q) .
$$

The flow of transactions from regular buyers is:

$$
t_{R}(q)=\gamma l(q)
$$

where $l(q)$ is the steady-steady number of regular buyers of a seller offering $q$. The number of regular buyers per seller offering $q$ is:

$$
l(q)=\frac{(B-\bar{n}) G^{\prime}(q)}{S F^{\prime}(q)}
$$

where $\bar{n}$ is the number of unmatched buyers; $(B-\bar{n}) G^{\prime}(q)$ is the number of buyers who are matched with a seller offering $q$; and $S F^{\prime}(q)$ is the number of sellers offering quality $q$.

We now determine the number of unmatched buyers and their type distribution. In steady state, the flow of buyers from the unmatched to the matched state must equal the flow out of the matched state and into the unmatched state. Let $n(R)$ denote the number of buyers who are unmatched and whose type is less than $R$. The total number of unmatched buyers is, thus, given by $n(\bar{R}) \equiv \bar{n}$.

An unmatched buyer of type $R$ becomes matched after transacting with a seller who offers abovereservation quality, which occurs at rate $\alpha_{B}(\theta)(1-F(R))$. A matched buyer exits the matched state when his match is exogenously destroyed, which occurs at rate $\delta$. As a result, in steady state, the following holds:

$$
n^{\prime}(R) \alpha_{B}(\theta)(1-(F(R)))=\delta\left(B H^{\prime}(R)-n^{\prime}(R)\right) \Rightarrow n^{\prime}(R)=\frac{\delta B H^{\prime}(R)}{\delta+\alpha_{B}(\theta)(1-F(R))},
$$

which we can rewrite as:

$$
n(R)=\int_{\underline{R}}^{R} \frac{B \delta}{\delta+\alpha_{B}(\theta)(1-F(x))} d H(x) .
$$

Therefore, we have:

$$
\begin{aligned}
\bar{n} & =\int_{\underline{R}}^{\bar{R}} \frac{B \delta}{\delta+\alpha_{B}(\theta)(1-F(x))} d H(x) \\
B-\bar{n} & =B\left(1-\int_{\underline{R}}^{\bar{R}} \frac{\delta}{\delta+\alpha_{B}(\theta)(1-F(x))} d H(x)\right)=\int_{\underline{R}}^{\bar{R}} \frac{B \alpha_{B}(\theta)(1-F(x))}{\delta+\alpha_{B}(\theta)(1-F(x))} d H(x) .
\end{aligned}
$$

We now characterize $G(\cdot)$. The mass of matched buyers receiving quality up to $q$ is $(B-\bar{n}) G(q)$. An unmatched type- $R$ buyer flows into this group if $R \leq q$ and he samples a seller who offers quality less than $q$, which occurs at rate $\alpha_{B}(\theta)(F(q)-F(R))$. A buyer flows out of this group if the match is exogenously destroyed or if he samples a new seller whose quality if greater than $q$, which occurs at rate $\delta+\alpha_{B}(\theta)(1-F(q))$. Equating these flows yields:

$$
\begin{gathered}
\alpha_{B}(\theta) \int_{\underline{R}}^{q}(F(q)-F(R)) d n(R)=(B-\bar{n}) G(q)\left(\delta+\alpha_{B}(\theta)(1-F(q))\right), \\
\Rightarrow(B-\bar{n}) G(q) \frac{\alpha_{B}(\theta) B \delta \int_{\underline{R}}^{q} \frac{F(q)-F(x)}{\delta+\alpha_{B}(\theta)(1-F(x))} d H(x)}{\delta+\alpha_{B}(\theta)(1-F(q))} .
\end{gathered}
$$


Rearranging, we obtain:

$$
(B-\bar{n}) G^{\prime}(q)=\frac{\alpha_{B}(\theta) B \delta F^{\prime}(q) H(q)}{\left(\delta+\alpha_{B}(\theta)(1-F(q))\right)^{2}},
$$

which implies that the flow of transactions from regular buyers is:

$$
t_{R}(q)=\frac{\gamma \alpha_{B}(\theta) \theta \delta H(q)}{\left(\delta+\alpha_{B}(\theta)(1-F(q))\right)^{2}} .
$$

Combining results completes the proof of the Proposition.

We characterize the distribution of offered qualities, $F(\cdot)$ and the number of sellers who enter the market, taking as given the number of buyers $B$ and the distribution of their reservation values $H(\cdot)$.

Lemma 14 Consider sellers 1 and 2 with costs $c_{1}$ and $c_{2}$, and denote their actions by $q_{1}$ and $q_{2}$ and their profits by $\pi_{1}$ and $\pi_{2}$. Then: $c_{1}>c_{2} \Rightarrow q_{2}>q_{1}$ and $c_{1}>c_{2} \Rightarrow \pi_{2}>\pi_{1}$.

Proof. The proof for qualities is by contradiction. Suppose that $c_{1}>c_{2}$ and $q_{2} \leq q_{1}$. Recall that profits are given by $\pi_{c}(q)=(p-c q) t(q)$. Seller 1 chooses $q_{1}$ over $q_{2}$ and seller 2 chooses $q_{2}$ over $q_{1}$. Therefore:

$$
\begin{aligned}
& \left(p-c_{1} q_{1}\right) t\left(q_{1}\right) \geq\left(p-c_{1} q_{2}\right) t\left(q_{2}\right) \quad \Rightarrow p\left(t\left(q_{1}\right)-t\left(q_{2}\right)\right) \geq c_{1}\left(t\left(q_{1}\right) q_{1}-t\left(q_{2}\right) q_{2}\right) \\
& \left(p-c_{2} q_{2}\right) t\left(q_{2}\right) \geq\left(p-c_{2} q_{1}\right) t\left(q_{1}\right) \Rightarrow p\left(t\left(q_{1}\right)-t\left(q_{2}\right)\right) \leq c_{2}\left(t\left(q_{1}\right) q_{1}-t\left(q_{2}\right) q_{2}\right) .
\end{aligned}
$$

which yields the desired contradiction, after noting that $c_{1}>c_{2}$.

Regarding profits, note that:

$$
\pi_{1}=\left(p-c_{1} q_{1} t\left(q_{1}\right)\right)<\left(p-c_{2} q_{1}\right) t\left(q_{1}\right) \leq\left(p-c_{2} q_{2}\right) t\left(q_{2}\right)=\pi_{2}
$$

which completes the proof.

We characterize the marginal seller type $c^{* *}$ and the lowest quality that is offered, $q$ (we know from the previous Lemma that $\underline{q}$ is offered by the $c^{* *}$-seller). Two conditions need to be satisfied: first, $\underline{q}$ must give higher profits to $c^{* *}$ than any other quality level; second, $\underline{q}$ must cover the seller's flow cost. The proposition summarizes the result.

Proposition 15 Given $B$ and $H(\cdot)$, there is a unique marginal seller type $c^{* *}$ such that sellers with $c \leq c^{* *}$ participate in the market and sellers with $c>c^{* *}$ do not. The marginal seller type is determined by the solution to:

$$
\alpha_{B}(\theta) \theta H\left(\underline{q}\left(c^{* *}\right)\right)\left(p-c \underline{q}\left(c^{* *}\right)\right)\left(1+\frac{\gamma \delta}{\left.\left(\delta+\alpha_{B}(\theta)\right)\right)^{2}}\right)=K_{S},
$$

where $\underline{q}(c)$ solves

$$
c H(\underline{q})=H^{\prime}(\underline{q})(p-c \underline{q}) .
$$

Proof. It is immediate from Lemma 14 that a marginal type exists; she offers the lowest quality level and her profits are equal to the entry cost $K_{S}$. The profits of a type- $c$ seller who offers the lowest quality are:

$$
\underline{\pi}_{c}(q)=\alpha_{S}(\theta) H(q)(p-c q)\left(1+\frac{\gamma \delta}{\left.\left(\delta+\alpha_{B}(\theta)\right)\right)^{2}}\right) .
$$


The optimal choice of the lowest quality is given by the root of:

$$
\underline{\pi}_{c}(q)=\alpha_{S}(\theta)\left(1+\frac{\gamma \delta}{\left.\left(\delta+\alpha_{B}(\theta)\right)\right)^{2}}\right)\left(H^{\prime}(q)(p-c q)-c H(q)\right) .
$$

The second derivative of profits is always negative due to the log-concavity of $H(\cdot)$ :

$$
\underline{\pi}_{c}^{\prime \prime}(q)=\alpha_{S}(\theta)\left(1+\frac{\gamma \delta}{\left.\left(\delta+\alpha_{B}(\theta)\right)\right)^{2}}\right)\left(H^{\prime \prime}(q)(p-c q)-2 c H(q)-c H^{\prime}(q)\right)<0 .
$$

Therefore, the optimal quality choice for a type- $c$ seller who offers the lowest quality $\underline{q}(c)$ is given by equation (C3).

The marginal seller type determines the buyer-seller ratio according to $\theta=\frac{B}{S \bar{D}\left(c^{* *}\right)}$. The profits of the seller who offers $\underline{q}(c)$ are decreasing in her cost $c$ :

$$
\frac{d \underline{\pi}_{c}(\underline{q}(c))}{d c}=\frac{\underline{\pi}_{c}(\underline{q})}{d \underline{q}} \underline{q}^{\prime}(c)+\frac{\partial \underline{\pi}_{c}(\underline{q}(c))}{\partial c}+\frac{\partial \underline{\pi}_{c}(\underline{q}(c))}{\partial \theta} \frac{d \theta}{d c}<0 .
$$

It is also immediate that:

$$
\begin{aligned}
& \lim _{c \rightarrow 0} \underline{\pi}(\underline{q}(c))>K_{S}, \\
& \lim _{c \rightarrow \infty} \underline{\pi}(\underline{q}(c))<K_{S} .
\end{aligned}
$$

Therefore, there is a unique marginal seller type $c^{* *}$ who is determined by equation $(\mathrm{C} 2)$.

Corollary 16 The number of sellers who participate in the market is $S=\bar{S} \bar{D}\left(c^{* *}\right)$. The distribution of their types is $D(c)=\frac{\bar{D}(c)}{\bar{D}\left(c^{* *}\right)}$.

We now determine sellers' optimal $q^{* *}(c)$ for $c<c^{* *}$.

Proposition 17 Given $H(\cdot)$ and $B$, the optimal quality choice for sellers of type $c$ is given by the solution to the differential equation

$$
q^{* *} \prime(c)=\frac{2 H\left(q^{* *}(c)\right)\left(p-c q^{* *}(c)\right) \gamma \delta \alpha_{B}(\theta) D^{\prime}(c)}{\left[H^{\prime}\left(q^{* *}(c)\right)\left(p-c q^{* *}(c)\right)-c H\left(q^{* *}(c)\right)\right]\left(\delta+\alpha_{B}(\theta) D(c)\right)\left[\left(\delta+\alpha_{B}(\theta) D(c)\right)^{2}+\gamma \delta\right]},
$$

where the initial condition $q^{* *}\left(c^{* *}\right)=\underline{q}\left(c^{* *}\right)$.

The distribution of qualities is:

$$
F(q)=1-D\left(q^{* *-1}(q)\right)
$$

Proof. To characterize the optimal quality offer $q^{* *}(c)$ for a type- $c$ seller with $c<c^{* *}$, we rewrite her profits as if she decides which other type $c^{\prime}$ to imitate rather than which quality to offer. In other words, her profits from offering some quality $q^{\prime}$ are written in terms of imitating type $c^{\prime}$, who offers quality $q^{\prime}=q^{* *}\left(c^{\prime}\right)$. We have:

$$
\pi_{c}\left(c^{\prime}\right)=\alpha_{S}(\theta) H\left(q^{* *}\left(c^{\prime}\right)\right)\left(p-c q^{* *}\left(c^{\prime}\right)\right)\left(1+\frac{\gamma \delta}{\left(\delta+\alpha_{B}(\theta) D\left(c^{\prime}\right)\right)^{2}}\right) .
$$

The advantage of formulating the choice in terms of $c^{\prime}$ rather on than $q^{\prime}$ is that the term in the denominator depends on the exogenous type distribution $D(\cdot)$ rather than on the endogenous quality distribution $F(\cdot)$. We can recover the quality distribution once we construct $q^{* *}(c)$. 
We differentiate profits with respect to $c^{\prime}$ :

$$
\begin{aligned}
\pi_{c}^{\prime}\left(c^{\prime}\right)= & \alpha_{S}(\theta)\left(H^{\prime}\left(q^{* *}\left(c^{\prime}\right)\right) q^{* *} \prime\left(c^{\prime}\right)\left(p-c q^{* *}\left(c^{\prime}\right)\right)\left(1+\frac{\gamma \delta}{\left(\delta+\alpha_{B}(\theta) D\left(c^{\prime}\right)\right)^{2}}\right)\right. \\
& -\frac{c q^{* *}\left(c^{\prime}\right) H\left(q^{* *}\left(c^{\prime}\right)\right) \gamma \delta}{\left(\delta+\alpha_{B}(\theta) D\left(c^{\prime}\right)\right)^{2}}-H\left(q^{* *}\left(c^{\prime}\right)\right)\left(p-c q^{* *}\left(c^{\prime}\right) \frac{\gamma \delta 2\left(\delta+\alpha_{B}(\theta) D\left(c^{\prime}\right)\right) D^{\prime}\left(c^{\prime}\right)}{\left(\delta+\alpha_{B}(\theta) D\left(c^{\prime}\right)\right)^{3}}\right) .
\end{aligned}
$$

Equating the derivative to zero and setting $c^{\prime}=c$ leads to equation (C4), which, together with the initial condition $q^{* *}\left(c^{* *}\right)=\underline{q}\left(c^{* *}\right)$, defines $q^{* *}(c)$ for $c \in\left(0, c^{* *}\right)$.

\section{C.3 Equilibrium}

The proof of equilibrium existence is identical to that of the baseline model and is, therefore, omitted. 
TABle D1: The Effect of Penalties, Higher Meeting Rate and Lower Destruction Rate

\begin{tabular}{|c|c|c|c|}
\hline & BASELINE & $\overline{\text { HIGHER } \gamma}$ & LOWER $\delta$ \\
\hline \multirow{2}{*}{ FRACTION OF RIP-OFFS (\%) } & 15.862 & 1.357 & 0.674 \\
\hline & {$[13.646 ; 16.812]$} & {$[1.273 ; 1.441]$} & {$[0.478 ; 0.770]$} \\
\hline \multirow{2}{*}{ Average Pure Grams per $\$ 100$} & 0.616 & 0.943 & 1.070 \\
\hline & {$[0.597 ; 0.636]$} & {$[0.933 ; 0.955]$} & {$[1.051 ; 1.095]$} \\
\hline \multirow{2}{*}{ St. Dev. Pure Grams per $\$ 100$} & 0.271 & 1.134 & 0.858 \\
\hline & {$[0.256 ; 0.279]$} & {$[1.103 ; 1.164]$} & {$[0.737 ; 0.902]$} \\
\hline \multirow{2}{*}{ Active Buyers, in Millions } & 3.431 & 1.043 & 1.083 \\
\hline & {$[3.312 ; 3.530]$} & {$[1.024 ; 1.053]$} & {$[1.046 ; 1.111]$} \\
\hline \multirow{2}{*}{ Active Sellers, in Millions } & 0.290 & 1.043 & 1.083 \\
\hline & {$[0.271 ; 0.295]$} & {$[1.024 ; 1.053]$} & {$[1.046 ; 1.111]$} \\
\hline \multirow{2}{*}{ Fraction of Matched Buyers (\%) } & 54.040 & 1.056 & 1.054 \\
\hline & {$[52.420 ; 55.100]$} & {$[1.029 ; 1.103]$} & {$[0.997 ; 1.080]$} \\
\hline \multirow{2}{*}{ Average Number of Purchases Per Month } & 12.726 & 1.046 & 1.227 \\
\hline & {$[12.228 ; 13.389]$} & {$[1.026 ; 1.076]$} & {$[1.176 ; 1.241]$} \\
\hline \multirow{2}{*}{ Average Pure Grams Consumed per Month } & 9.464 & 1.056 & 1.242 \\
\hline & {$[9.057 ; 9.990]$} & {$[1.034 ; 1.086]$} & {$[1.192 ; 1.260]$} \\
\hline
\end{tabular}

Notes-This table reports market outcomes in two counterfactual cases: 1) buyers' meeting rate with regular dealers $\gamma$ is 20-percent higher than in the baseline case; and 2) the destruction rate $\delta$ is 20percent lower than in the baseline case, expressed as ratios over the corresponding values in the baseline case. 95-percent confidence intervals in brackets.

\section{Additional Counterfactuals and Sensitivity Analyses}

We perform the following additional counterfactuals, with results displayed in Table D1: 1) a 20percent increase in $\gamma$; and 2) a 20-percent decrease in $\delta$. As in Section 5.4, we report quantitative results for the population (i.e., without sample selection) as ratios of the corresponding values in the baseline case (i.e., a ratio larger than one implies an increase relative to the baseline case).

To understand the results of Table D1, notice that an increase in $\gamma$ increases buyers' frequency of consumption which, in turn, increases their value of participating in the market. This leads to an increase in the entry of buyers, similarly to the counterfactual with lower $K_{B}$ (of course, sellers' incentives are also affected, but this turns out to be of second-order importance). As in that counterfactual, more buyers enter the market, rip-offs increase and quality dispersion increases. Since sellers' free entry condition, equation (A2), does not change, the buyer-seller ratio exactly equals that of the baseline case. Therefore, a larger number of sellers participate in the market than in the baseline case, as well.

A reduction in $\delta$ increases the value of a long-term relationship, as it leads to less frequent exogenous dissolution. This change increases the attractiveness for sellers of acquiring regular customers and, as a result, more sellers offer positive quality, rip-offs decline, average quality increases and quality dispersion drops. The reduction in rip-offs and exogenous match dissolution leads to an increase in the proportion of buyers who are matched, thereby increasing buyers' value of participating in the market and, thus, their numbers. Since again sellers' free entry condition, equation (A2), does not change, the buyer-seller ratio exactly equals that of the baseline case. Therefore, a larger number of sellers participate in the market than in the baseline case, as well. 
TABLE D2: Observable Quality: Urn-Ball Matching Function

\begin{tabular}{|c|c|c|c|}
\hline & BASELINE & $\begin{array}{l}\text { OBSERVABLE } q \text {, } \\
\text { PARTIAL EQ. }\end{array}$ & $\begin{array}{l}\text { OBSERVABLE } q \text {, } \\
\text { GENERAL EQ. }\end{array}$ \\
\hline \multirow{2}{*}{ FRACTION OF RIP-OFFS (\%) } & 15.862 & 0.000 & 0.000 \\
\hline & {$[13.646 ; 16.812]$} & {$[0.000 ; 0.000]$} & {$[0.000 ; 0.000]$} \\
\hline \multirow{2}{*}{ Average Pure Grams Per $\$ 100$} & 0.616 & 1.204 & 1.197 \\
\hline & {$[0.597 ; 0.636]$} & {$[1.160 ; 1.261]$} & {$[1.153 ; 1.217]$} \\
\hline \multirow{2}{*}{ St. Dev. Pure Grams Per $\$ 100$} & 0.271 & 0.184 & 0.168 \\
\hline & {$[0.256 ; 0.279]$} & {$[0.156 ; 0.287]$} & {$[0.144 ; 0.175]$} \\
\hline \multirow{2}{*}{ ACtive Buyers, in MiLlions } & 3.431 & 1.000 & 1.046 \\
\hline & {$[3.312 ; 3.530]$} & {$[1.000 ; 1.000]$} & {$[1.030 ; 1.063]$} \\
\hline \multirow{2}{*}{ ACtive Sellers, in Millions } & 0.290 & 1.000 & 0.820 \\
\hline & {$[0.271 ; 0.295]$} & {$[1.000 ; 1.000]$} & {$[0.806 ; 0.842]$} \\
\hline \multirow{2}{*}{ Fraction of Matched Buyers (\%) } & 54.040 & 1.054 & 0.962 \\
\hline & {$[52.420 ; 55.100]$} & {$[1.024 ; 1.109]$} & {$[0.955 ; 1.035]$} \\
\hline \multirow{2}{*}{ Average Number of Purchases Per Month } & 12.726 & 1.053 & 0.968 \\
\hline & {$[12.228 ; 13.389]$} & {$[1.040 ; 1.086]$} & {$[0.945 ; 0.990]$} \\
\hline \multirow{2}{*}{ Average Pure Grams Consumed Per Month } & 9.464 & 1.082 & 0.988 \\
\hline & {$[9.057 ; 9.990]$} & {$[1.065 ; 1.115]$} & {$[0.967 ; 1.008]$} \\
\hline
\end{tabular}

Notes-This table reports market outcomes in the counterfactual cases in which buyers can observe drugs' purity before purchasing, expressed as ratios over the corresponding values in the baseline case. 95-percent confidence intervals in brackets.

Moreover, we perform several sensitivity analyses to the counterfactuals of Section 5.4. Overall, these additional analyses deliver results that are very similar results to those reported in Section 5.4, thus showing their robustness. More specifically:

- We perform our counterfactuals on observable quality and on penalties using alternative matching functions. The counterfactuals of Section 5.4 impose a Cobb-Douglas matching function. Tables D2 and D3 display the results obtained with the urn-ball matching function between $B$ buyers and $S$ sellers: $M(B, S)=\omega_{1} S\left(1-e^{-\omega_{2} \frac{B}{S}}\right)$, where we set the parameters $\omega_{1}$ and $\omega_{2}$ to match the estimate of $\alpha_{B}$ reported in Table 2 and the average number of transactions per seller reported in Section 5.3. Tables D4 and D5 display the results obtained with the telegraph line: $M(B, S)=$ $\frac{\omega_{3} B S}{\omega_{4} B+S}$, where we set the parameters $\omega_{3}$ and $\omega_{4}$ to match the estimate of $\alpha_{B}$ reported in Table 2 and the average number of transactions per seller reported in Section 5.3. Note that these alternative matching functions have obviously no effect in the observable- $q$, partial-equilibrium case, as the the numbers of buyers and of sellers are fixed at their baseline values.

- We perform our counterfactuals on penalties using alternative values of sellers' and buyers' fixed costs. Tables D6-D7 display the results in the cases in which we decrease sellers' and buyers' costs by 10 percent and 20 percent, respectively. 
TABLE D3: The Effect of Penalties: Urn-Ball Matching Function

\begin{tabular}{|c|c|c|c|}
\hline & BASELINE & LOWER $K_{S}$ & LOWER $K_{B}$ \\
\hline \multirow{2}{*}{ FRACTION OF RIP-OFFS (\%) } & 15.862 & 2.481 & 1.162 \\
\hline & {$[13.646 ; 16.812]$} & {$[2.374 ; 2.841]$} & {$[1.080 ; 1.209]$} \\
\hline \multirow{2}{*}{ Average Pure Grams per $\$ 100$} & 0.616 & 0.712 & 0.972 \\
\hline & {$[0.597 ; 0.636]$} & {$[0.684 ; 0.726]$} & {$[0.950 ; 0.983]$} \\
\hline \multirow{2}{*}{ St. Dev. Pure Grams Per $\$ 100$} & 0.2 & 1.3 & 1.0 \\
\hline & {$[0.256$; } & {$[1.282 ;$} & {$[1.029 ;$} \\
\hline \multirow{2}{*}{ Active Buyers, in Millions } & 3.43 & 0.867 & 1.036 \\
\hline & {$[3.312$} & {$[0.850$} & {$[1.008 ; 1.042]$} \\
\hline \multirow{2}{*}{ Active Sellers, in Millions } & 0.290 & 1.565 & 1.036 \\
\hline & {$[0.271 ; 0.295]$} & {$[1.552 ; 1.636]$} & {$[1.008 ; 1.042]$} \\
\hline \multirow{2}{*}{ FRACTION OF MATCHED BUYERS (\%) } & 54.0 & 1.001 & 0.996 \\
\hline & {$[52.420$} & {$[0.971 ; 1.051]$} & {$[0.955 ; 1.008]$} \\
\hline \multirow{2}{*}{ Average Number of Purchases Per Month } & 12.7 & 1.0 & 0.991 \\
\hline & [12.228; & {$[1.058 ; 1.125]$} & {$[0.971 ; 1.015]$} \\
\hline \multirow{2}{*}{ Average Pure Grams Consumed per Month } & 9.464 & 1.022 & 0.988 \\
\hline & {$[9.057 ; 9.990]$} & {$[1.005 ; 1.072]$} & {$[0.969 ; 1.016]$} \\
\hline
\end{tabular}

Notes-This table reports market outcomes in the counterfactual cases in which buyers' cost $K_{B}$ and sellers' cost $K_{S}$ are 15-percent lower than in the baseline case, respectively, expressed as ratios over the corresponding values in the baseline case. 95-percent confidence intervals in brackets.

TABle D4: Observable Quality: Telegraph-Line Matching Function

\begin{tabular}{|c|c|c|c|}
\hline & BASELINE & $\begin{array}{l}\text { OBSERVABLE } q, \\
\text { PARTIAL EQ. }\end{array}$ & $\begin{array}{l}\text { OBSERVABLE } q, \\
\text { GENERAL EQ. }\end{array}$ \\
\hline \multirow{2}{*}{ FrACTION OF RIP-OFFS (\%) } & 15.862 & 0.000 & 0.000 \\
\hline & {$[13.646 ; 16.812]$} & {$[0.000 ; 0.000]$} & {$[0.000 ; 0.000]$} \\
\hline \multirow{2}{*}{ Average Pure Grams per $\$ 100$} & 0.616 & 1.204 & 1.197 \\
\hline & {$[0.597 ; 0.636]$} & {$[1.160 ; 1.261]$} & {$[1.165 ; 1.216]$} \\
\hline \multirow{2}{*}{ St. Dev. Pure Grams Per $\$ 100$} & 0.271 & 0.184 & 0.170 \\
\hline & {$[0.256 ; 0.279]$} & {$[0.156 ; 0.287]$} & {$[0.142 ; 0.180]$} \\
\hline \multirow{2}{*}{ Active Buyers, in Millions } & 3.431 & 1.000 & 1.057 \\
\hline & {$[3.312 ; 3.530]$} & {$[1.000 ; 1.000]$} & {$[1.037 ; 1.074]$} \\
\hline \multirow{2}{*}{ ACtive Sellers, in Millions } & 0.290 & 1.000 & 0.822 \\
\hline & {$[0.271 ; 0.295]$} & {$[1.000 ; 1.000]$} & {$[0.807 ; 0.843]$} \\
\hline \multirow{2}{*}{ Fraction of Matched Buyers (\%) } & 54.040 & 1.054 & 0.997 \\
\hline & {$[52.420 ; 55.100]$} & {$[1.024 ; 1.109]$} & {$[0.955 ; 1.037]$} \\
\hline \multirow{2}{*}{ Average Number of Purchases per Month } & 12.726 & 1.053 & 0.981 \\
\hline & {$[12.228 ; 13.389]$} & {$[1.040 ; 1.086]$} & {$[0.960 ; 1.005]$} \\
\hline \multirow{2}{*}{ Average Pure Grams Consumed Per Month } & 9.464 & 1.082 & 1.004 \\
\hline & {$[9.057 ; 9.990]$} & {$[1.065 ; 1.115]$} & {$[0.979 ; 1.029]$} \\
\hline
\end{tabular}

Notes-This table reports market outcomes in the counterfactual cases in which buyers can observe drugs' purity before purchasing, expressed as ratios over the corresponding values in the baseline case. 95-percent confidence intervals in brackets. 
Table D5: The Effect of Penalties: Telegraph-Line Matching Function

\begin{tabular}{|c|c|c|c|}
\hline & 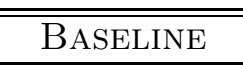 & LOWER $K_{S}$ & LOWER $K_{B}$ \\
\hline \multirow{2}{*}{ FRACTION OF RIP-OFFS (\%) } & 15.862 & 2.053 & 1.162 \\
\hline & {$[13.646 ; 16.812]$} & {$[1.956 ; 2.281]$} & {$[1.082 ; 1.211]$} \\
\hline \multirow{2}{*}{ Average Pure Grams Per $\$ 100$} & 0.616 & 0.798 & 0.972 \\
\hline & {$[0.597 ; 0.636]$} & {$[0.777 ; 0.806]$} & {$[0.948 ; 0.982]$} \\
\hline \multirow{2}{*}{ St. Dev. Pure Grams per $\$ 100$} & 0.2 & 1.2 & 1.062 \\
\hline & {$[0.256$} & {$[1.235 ;$} & {$[1.028 ; 1$} \\
\hline \multirow{2}{*}{ Active Buyers, in Millions } & 3.4 & 0.915 & 1.036 \\
\hline & {$[3.312$} & {$[0.892 ;$} & {$[1.002 ; 1.040]$} \\
\hline \multirow{2}{*}{ Active Sellers, in Millions } & $0.2 !$ & 1.415 & 1.036 \\
\hline & {$[0.271 ; 0.295]$} & {$[1.392 ; 1.453]$} & {$[1.002 ; 1.040]$} \\
\hline \multirow[t]{2}{*}{ Fraction of Matched Buyers (\%) } & 54.040 & 0.986 & 0.996 \\
\hline & [52.420; 55.100] & {$[0.931 ; 1.037]$} & {$[0.939 ; 1.016]$} \\
\hline Average Number of Purchases per Month & 12.726 & 1.05 & $\begin{array}{c}0.991 \\
{[0.965: 1.012]}\end{array}$ \\
\hline \multirow{2}{*}{ Average Pure Grams Consumed per Month } & 9.464 & 1.025 & 0.988 \\
\hline & {$[9.057 ; 9.990]$} & {$[0.981 ; 1.047]$} & {$[0.961 ; 1.014$} \\
\hline
\end{tabular}

Notes-This table reports market outcomes in the counterfactual cases in which buyers' cost $K_{B}$ and sellers' cost $K_{S}$ are 15-percent lower than in the baseline case, respectively, expressed as ratios over the corresponding values in the baseline case. 95-percent confidence intervals in brackets.

TABle D6: The Effect of Penalties, Alternative Costs I

\begin{tabular}{|c|c|c|c|}
\hline & BASELINE & LOWER $K_{S}$ & LOWER $K_{B}$ \\
\hline \multirow{2}{*}{ FRACTION OF RIP-OFFS (\%) } & 15.862 & 1.445 & 1.109 \\
\hline & {$[13.646 ; 16.812]$} & {$[1.399 ; 1.545]$} & {$[1.058 ; 1.141]$} \\
\hline \multirow{2}{*}{ Average Pure Grams per $\$ 100$} & 0.61 & 0.906 & 0.980 \\
\hline & {$[0.597 ; 0.636]$} & {$[0.898 ; 0.916]$} & {$[0.963 ; 0.988]$} \\
\hline \multirow{2}{*}{ St. Dev. Pure Grams Per $\$ 100$} & 0.2 & 1.134 & 1.0 \\
\hline & {$[0.256$} & {$[1.125$} & {$[1.017$} \\
\hline \multirow{2}{*}{ Active Buyers, in Millions } & & & \\
\hline & {$[3.312$; } & {$[0.954 ; 0.972]$} & {$[1.000 ; 1.029]$} \\
\hline \multirow{2}{*}{ Active Sellers, in Millions } & 0.2 & 1.180 & 1.024 \\
\hline & {$[0.271$} & {$[1.178 ; 1.200]$} & {$[1.000 ; 1.029]$} \\
\hline \multirow[t]{2}{*}{ FRACTION OF MATCHED BUYERS (\%) } & 54.040 & 0.991 & 0.979 \\
\hline & $\begin{array}{l}{[52.420 ; 55.100]} \\
12.726\end{array}$ & $\begin{array}{c}{[0.967 ; 1.026]} \\
1.011\end{array}$ & $\begin{array}{c}{[0.959 ; 1.027]} \\
0.988\end{array}$ \\
\hline Average Number of Purchases per Month & {$[12.228 ; 1$} & {$[0.988 ; 1.036]$} & {$[0.966 ; 1.019]$} \\
\hline Average Pure Grams Consumed per Month & $\begin{array}{c}9.464 \\
0.057 \cdot 0.00\end{array}$ & 0.995 & 0.987 \\
\hline
\end{tabular}

Notes - This table reports market outcomes in the counterfactual cases in which buyers' cost $K_{B}$ and sellers' cost $K_{S}$ are 10-percent lower than in the baseline case, respectively, expressed as ratios over the corresponding values in the baseline case. 95-percent confidence intervals in brackets. 
TABle D7: The Effect of Penalties, Alternative Costs II

\begin{tabular}{|c|c|c|c|}
\hline & BASELINE & LOWER $K_{S}$ & LOWER $K_{B}$ \\
\hline \multirow{2}{*}{ FRACTION OF RIP-OFFS (\%) } & 15.862 & 1.890 & 1.211 \\
\hline & {$[13.646 ; 16.812]$} & {$[1.791 ; 2.073]$} & {$[1.107 ; 1.276]$} \\
\hline \multirow{2}{*}{ Average Pure Grams Per $\$ 100$} & 0.616 & 0.823 & 0.962 \\
\hline & {$[0.597 ; 0.636]$} & {$[0.808 ; 0.838]$} & {$[0.953 ; 0.977]$} \\
\hline \multirow{2}{*}{ St. Dev. Pure Grams Per $\$ 100$} & 0.271 & 1.233 & 1.078 \\
\hline & {$[0.256 ; 0.279]$} & {$[1.202 ; 1.299]$} & {$[1.038 ; 1.101]$} \\
\hline \multirow{2}{*}{ ACtive Buyers, in Millions } & 3.431 & 0.916 & 1.047 \\
\hline & {$[3.312 ; 3.530]$} & {$[0.902 ; 0.943]$} & {$[1.024 ; 1.059]$} \\
\hline \multirow{2}{*}{ ACtive SELLERS, in Millions } & 0.290 & 1.432 & 1.047 \\
\hline & {$[0.271 ; 0.295]$} & {$[1.409 ; 1.474]$} & {$[1.024 ; 1.059]$} \\
\hline \multirow{2}{*}{ Fraction of MATChED BuYERS (\%) } & 54.040 & 0.994 & 0.980 \\
\hline & {$[52.420 ; 55.100]$} & {$[0.955 ; 1.043]$} & {$[0.952 ; 1.005]$} \\
\hline \multirow{2}{*}{ Average Number of Purchases Per Month } & 12.726 & 1.043 & 0.986 \\
\hline & {$[12.228 ; 13.389]$} & {$[1.014 ; 1.060]$} & {$[0.960 ; 1.012]$} \\
\hline \multirow[t]{2}{*}{ Average Pure Grams Consumed Per Month } & 9.464 & 1.012 & 0.984 \\
\hline & {$[9.057 ; 9.990]$} & {$[0.984 ; 1.031]$} & {$[0.956 ; 1.009]$} \\
\hline
\end{tabular}

Notes - This table reports market outcomes in the counterfactual cases in which buyers' cost $K_{B}$ and sellers' cost $K_{S}$ are 20-percent lower than in the baseline case, respectively, expressed as ratios over the corresponding values in the baseline case. 95-percent confidence intervals in brackets. 